\title{
Differential abundances of open clusters and their tidal tails: Chemical tagging and chemical homogeneity $\star, \star \star$
}

\author{
L. Casamiquela ${ }^{1}$, Y. Tarricq ${ }^{1}$, C. Soubiran ${ }^{1}$, S. Blanco-Cuaresma ${ }^{2}$, P. Jofré ${ }^{3}$, U. Heiter ${ }^{4}$, and M. Tucci Maia ${ }^{3}$ \\ ${ }^{1}$ Laboratoire d'Astrophysique de Bordeaux, Univ. Bordeaux, CNRS, B18N, allée Geoffroy Saint-Hilaire, 33615 Pessac, France \\ e-mail: laia.casamiquela-floriach@u-bordeaux.fr \\ 2 Harvard-Smithsonian Center for Astrophysics, Cambridge, MA 02138, USA \\ 3 Núcleo de Astronomía, Facultad de Ingeniería y Ciencias, Universidad Diego Portales, Av. Ejército 441, Santiago, Chile \\ ${ }^{4}$ Observational Astrophysics, Department of Physics and Astronomy, Uppsala University, Box 516, 75120 Uppsala, Sweden
}

Received 23 October 2019 / Accepted 17 December 2019

\begin{abstract}
Context. Well studied open clusters (OCs) of the solar neighborhood are frequently used as reference objects to test galactic and stellar theories. For that purpose, their chemical composition needs to be known with a high level of confidence. It is also important to clarify if each OC is chemically homogeneous and if it has a unique chemical signature.

Aims. The aims of this work are (1) to determine accurate and precise abundances of 22 chemical species (from $\mathrm{Na}$ to $\mathrm{Eu}$ ) in the Hyades, Praesepe, and Rupecht 147 by using a large number of stars at different evolutionary states, (2) to evaluate the level of chemical homogeneity of these OCs, and (3) to compare their chemical signatures.

Methods. We gathered $\sim 800$ high resolution and high signal-to-noise spectra of $\sim 100$ members in the three clusters, which were obtained with the latest memberships based on Gaia DR2 data. We built a pipeline, which computes atmospheric parameters and strictly line-by-line differential abundances among twin stars in our sample. With this method, we were able to reach a very high precision in the abundances (0.01-0.02 dex in most of the elements).

Results. We find large differences in the absolute abundances in some elements, which can be attributed to diffusion, non-local thermodynamic equilibrium (non-LTE) effects, or systematics in the analysis. For the three OCs, we find strong correlations in the differential abundances between different pairs of elements. According to our experiment with synthetic data, this can be explained by some level of chemical inhomogeneity. We compare differential abundances of several stars from the Hyades and Praesepe tails: The stars that differ more in chemical abundances also have distinct kinematics, even though they have been identified as members of the tail.

Conclusions. It is possible to obtain high precision abundances using a differential analysis even when mixing spectra from different instruments. With this technique, we find that the Hyades and Preasepe have the same chemical signature when $\mathrm{G}$ dwarfs and $\mathrm{K}$ giants are considered. Despite a certain level of inhomogeneity in each cluster, it is still possible to clearly distinguish the chemical signature of the older cluster Ruprecht 147 when compared to the Hyades and Praesepe.
\end{abstract}

Key words. stars: abundances - techniques: spectroscopic - open clusters and associations: individual: NGC 2632 open clusters and associations: individual: Hyades - open clusters and associations: individual: Ruprecht 147

\section{Introduction}

Open clusters (OCs) of different ages and chemical compositions are ideal to perform tests on star formation and evolution theories, and they have long been used to better understand the history of the Galactic disk. Several spectroscopic surveys dedicate a significant observing time to OCs, such as the Gaia-ESO survey (Gilmore et al. 2012; Randich \& Gilmore 2013), Apache Point Observatory Galactic Evolution Experiment (APOGEE, Majewski et al. 2017), and Open Cluster Chemical Abundances from Spanish Observatories (OCCASO, Casamiquela et al. 2019), among others. In these surveys, OCs provide fundamental material for calibrating the stellar parameters, in particular, the dependencies of abundances as a function of stellar parameters (Jofré et al. 2019).

* Full Tables 2, A.1-A.3 are only available at the CDS via anonymous ftp to cdsarc.u-strasbg.fr $(130.79 .128 .5)$ or via http: //cdsarc.u-strasbg.fr/viz-bin/cat/J/A+A/635/A8

$\star \star$ Thanks to observations at Telescope Bernard Lyot and data retrieved from the archives: ESO, TNG, FIES, ELODIE, ESPaDOnS and NARVAL.
OCs have long been thought to be chemically homogeneous (e.g., Friel et al. 2002) as a result of the hypothesis that the cloud from which the cluster was formed was uniformly mixed. Observed abundance dispersions are typically around $0.05 \mathrm{dex}$, which is usually at the same level of the measurement uncertainties. By using a strictly line-by-line differential analysis method, such uncertainties can be lowered to better assess the homogeneity of OCs. Differential chemical abundance analysis has been mainly used to analyze abundance variations among solar twins (e.g., Meléndez et al. 2009; Nissen 2015; Tucci Maia et al. 2016; Mahdi et al. 2016). Applications in other contexts can also be found, such as studies of nearby stars, globular or open clusters, and benchmark stars (e.g., Heiter \& Luck 2003; Yong et al. 2013; Önehag et al. 2014; Jofré et al. 2015; Hawkins et al. 2016; Liu et al. 2019). By using a reference star with stellar parameters close to the program stars, this method allows one to reach a very high precision in abundances, on the order of 0.01 dex, because it minimizes the uncertainty coming from errors in the characterization of spectral lines. In particular, Liu et al. (2016a) and Spina et al. (2018) analyzed 16 and five solar analogs, which are members of the Hyades and the Pleiades, 
Table 1. Properties of the OCs studied in this work.

\begin{tabular}{lrccccc}
\hline \hline \multirow{2}{*}{ Cluster } & $D[\mathrm{pc}]$ & \multicolumn{4}{c}{$\log (\mathrm{age}[\mathrm{yr}])$} & \multirow{2}{*}{\begin{tabular}{c} 
Num. \\
\cline { 3 - 6 }
\end{tabular}} \\
\cline { 3 - 6 } & & D02 & K13 & GC18 & B19 & stars \\
\hline Hyades & 48 & 8.90 & - & 8.90 & - & 62 \\
NGC 2632 & 186 & 8.86 & 8.92 & 8.85 & 8.87 & 22 \\
Rup 147 & 284 & 9.40 & 9.33 & 9.30 & - & 24 \\
\hline
\end{tabular}

Notes. We indicate the cluster distance $D$ (from Gaia DR2 parallaxes, Gaia Collaboration 2018a) and the ages from Dias et al. (2002, D02), Kharchenko et al. (2005, K13), Gaia Collaboration (2018a, GC18), and Bossini et al. (2019, B19). The number of stars with high resolution spectra is given in the rightmost column.

respectively, to show chemical inhomogeneities at the level of 0.02 dex.

In this paper, we investigate how the line-by-line differential method can be applied to stars over a larger range of evolutionary states. This is important because distant $\mathrm{G}$ dwarfs are usually not observable at high spectral resolution because they are too faint to obtain high signal-to-noise ratio $(\mathrm{S} / \mathrm{N})$ spectra with current spectrographs. We thus propose that intrinsically brighter stars, such as clump giants and F dwarfs in nearby OCs, be used as reference objects for abundance studies involving distant targets. By measuring differential abundances with respect to stars of a similar evolutionary state in local OCs, we expect to see subtle variations of chemical composition in the OC population. This methodology is also well suited to further develop the concept of chemical tagging that aims to identify stars of a common origin through their abundances. With our high precision differential abundances, we also measured the level of chemical homogeneity in OCs.

As our first step, we focus on three nearby objects, the Hyades, NGC 2632 (Praesepe), and Ruprecht 147, in order to establish a list of benchmark OCs with well-characterized chemical signatures. The Hyades and NGC 2632 have been considerably observed in the past (e.g., Gebran et al. 2010; Boesgaard et al. 2013; Gossage et al. 2018, among many others). Both appear to be similar in age, between 600 and $800 \mathrm{Myr}$, and have a metallicity of $\sim+0.15$ dex. Many high-quality spectra are available in public archives for further analyses. Surprisingly, Ruprecht 147 has been observed less, even though it is very interesting as it is the nearest OC that is older than 1 Gyr. One recent study provides an analysis of its chemical composition (Bragaglia et al. 2018) after Gaia DR2, retrieving a solar metallicity. The three clusters have clump giants which are excellent targets for spectroscopy because of their brightness and sharp lines allowing precise radial velocity and abundance determinations. Their population of FGK dwarfs is also easily observable at high resolution with 2-4 m class telescopes. In this study, we take advantage of new assessments of membership probabilities for stars in the fields of these clusters, which dramatically improved thanks to Gaia DR2 (Gaia Collaboration 2018b). With spectra from our own observations and from public archives, we provide high precision absolute and differential abundances for an unprecedented number of stars in each cluster up to large distances from the cluster's center, including their extended halo and tidal tails.

The paper is organized as follows. The selection of the target stars and the observational material is in Sect. 2, the method used and the explanation of the pipeline used to perform all computations is detailed in Sect. 3, the membership refinement using total Galactic velocities is explained in Sect. 4. Section 5 includes the results of the following spectroscopic analysis: the atmospheric parameters and chemical abundances of the cluster stars and the analyzed Gaia FGK benchmark stars (GBS). In Sect. 6 we detail the computation of the differential chemical abundances and we study their precision, the chemical signature of the stars in the tidal tails of the clusters, the homogeneity of the three clusters, and the possibility of chemical tagging.

\section{Observational material}

\subsection{Cluster and star selection from Gaia DR2}

As a starting point, we used the memberships lists provided for known OCs by Gaia Collaboration (2018a) and Cantat-Gaudin et al. (2018), who made use of Gaia DR2 astrometry. We focus on three nearby evolved OCs for which we gathered many high-resolution spectra of stars at different evolutionary stages, either from our own observations or from public archives: the Hyades (Melotte 25), Praesepe (NGC 2632), and Ruprecht 147. Their distances, ages, and the number of stars for which highresolution spectra are available are listed in Table 1.

The stars to be spectroscopic targets were selected according to the available membership information and their positions in the color-magnitude diagram. First we used the list of members from Cantat-Gaudin et al. (2018) and Gaia Collaboration (2018a) without any restrictions on the membership probability. As part of our targets, we included the stars found as members of the Hyades tails (Röser et al. 2019; Meingast \& Alves 2019) and the NGC 2632 tails (Röser \& Schilbach 2019). In the case of NGC 2632 and Ruprecht 147, we added stars (up to a distance $d \sim 150 \mathrm{pc}$ from the center), which have $(U, V, W)$ values ${ }^{1}$ that are compatible with the cluster (up to $2.5 \mathrm{~km} \mathrm{~s}^{-1}$ w.r.t. the mean cluster velocity), following the methodology of Meingast \& Alves (2019). In the case of NGC 2632 and Ruprecht 147, we also used Gaia DR2 information on radial velocity. We discarded those stars that had a radial velocity that was different by more than $1.2 \mathrm{MAD}^{2}$ from the median value of all stars. The stars without radial velocity information were kept. Second, for a precise spectroscopic characterization with our method, we require giant stars or dwarfs with temperatures within the range $6500 \mathrm{~K} \lesssim T_{\text {eff }} \lesssim 5000 \mathrm{~K}$. Hotter dwarfs usually have higher rotational velocities depending on age (Nielsen et al. 2013), and cooler stars have more crowded spectra due to molecular bands, giving less precise abundances. We used the PARSEC (Bressan et al. 2012) isochrones to determine the color range in the Gaia bands that correspond to these temperature limits $(0.6 \lesssim B p-R p \lesssim 1.1)$. Finally, dwarfs that were clearly located out of the main sequence in the color-absolute magnitude diagram were excluded to avoid binaries or possible contamination by nonmembers.

\subsection{Spectra}

Using the selection criteria described in the previous subsection, we selected more than 467 candidate stars to be studied spectroscopically. These close clusters have been studied by previous authors, and so we expected to find spectroscopic data for a large fraction of the selected stars. We queried the available public archives and searched for high resolution spectra $(R=\lambda / \Delta \lambda \gtrsim 45000)$. We did not put any restrictions on the $\mathrm{S} / \mathrm{N}$, a priori, so in many cases, we retrieved low $\mathrm{S} / \mathrm{N}$ spectra of the same star and instrument, which could then be coadded to reach a $\mathrm{S} / \mathrm{N}$ of $\sim 50$.

\footnotetext{
1 Computed using Gaia DR2 radial velocities.

2 Median Absolute Deviation.
} 
We retrieved, reduced, and calibrated spectra from the instruments as follows ${ }^{3}$.

- Ultraviolet and Visual Echelle Spectrograph (UVES) is a cross-dispersed echelle spectrograph that is installed at the second Very Large Telescope (VLT) unit, at the Paranal Observatory. It covers part of the optical spectral region with a resolution of $R \sim 45000$ or above, depending on the setup. We selected the setups according to the wavelength range of our line list for those centered on 580 and $564 \mathrm{~nm}$. We retrieved the spectra using the ESO Phase 3 spectral data webpage ${ }^{4}$.

- Fiber-fed Extended Range Optical Spectrograph (FEROS) is a high resolution $(R \sim 48000)$ echelle spectrograph that almost provides complete spectral coverage from 350 to $920 \mathrm{~nm}$. It is installed at the $2.2 \mathrm{~m} \mathrm{MPG/ESO}$ telescope at the La Silla Observatory. We retrieved the spectra by also using the ESO Phase 3 archive.

- High Accuracy Radial velocity Planet Searcher (HARPS) is a very high-resolution spectrograph $(R \sim 115000)$ that is installed at the $3.6 \mathrm{~m}$ telescope at the La Silla Observatory. The instrument was designed to obtain very high accuracy in radial velocity. The spectral range covered is $380-690 \mathrm{~nm}$. We retrieved the spectra by also using the ESO Phase 3 archive.

- HARPS-N is an instrument with very similar capabilities as HARPS $(R \sim 115000,380-690 \mathrm{~nm})$. It is attached to the Telescopio Nazionale Galileo (TNG) at the Observatory El Roque de Los Muchachos. We used the TNG archive ${ }^{5}$ to retrieve the data.

- FIbre-fed Echelle Spectrograph (FIES) is a cross-dispersed high-resolution echelle spectrograph with a spectral resolution of $R \sim 67000$ and a spectral range coverage from 370 to $910 \mathrm{~nm}$. It is attached to the Nordic Optical Telescope (NOT) at the Observatory El Roque de Los Muchachos. We used an exhaustive list of the public FIES spectra provided by the staff to cross-match them with our target stars and retrieve the spectra.

- Echelle SpectroPolarimetric Device for the Observation of Stars $(\mathrm{ESPaDOnS})$ is a high resolution $(R \sim 68000-81000$, depending on the configuration) spectropolarimeter that covers a spectral range of $370-1050 \mathrm{~nm}$. It is mounted at the CanadaFrench-Hawaii Telescope (CFHT) at the Mauna Kea Observatory. We cross-matched our targets with a list of ESPaDOns public observations provided by the staff to retrieve the reduced spectra.

- NARVAL is identical to ESPaDOnS. It is installed at the Telescope Bernard Lyot (TBL), atop the Pic du Midi observatory. We used the same strategy as for ESPaDOnS to retrieve the spectra.

- ELODIE was an echelle spectrograph $(R \sim 42000,390-$ $680 \mathrm{~nm}$ ) installed at the Observatoire de Haute-Provence (OHP) $1.93 \mathrm{~m}$ telescope until 2006. We used its dedicated archive ${ }^{6}$.

Additionally, we performed our own observing programs with NARVAL during two semesters (2018B and 2019A), during which we observed a total of 25 stars. In total, we collected 848 spectra corresponding to 108 different stars: 62, 22, and 24 from the Hyades, NGC 2632, and Ruprecht 147, respectively. In the cases where different spectra from the same instrument

\footnotetext{
3 We also retrieved spectra from the SOPHIE archive. However, we encountered several problems in recovering atmospheric parameters from SOPHIE spectra, and hence we did not include them in this work. 4 http://archive.eso.org/wdb/wdb/adp/phase3_spectral/ form

5 The archive (http://archives.ia2.inaf.it/tng/faces/ search . xhtml?dswid=-9619) does not allow for an automatic search for a large number of stars, so we queried the stars of Rup 147 that we knew had been observed by Bragaglia et al. (2018) in advance.

6 http://atlas.obs-hp.fr/elodie/
}

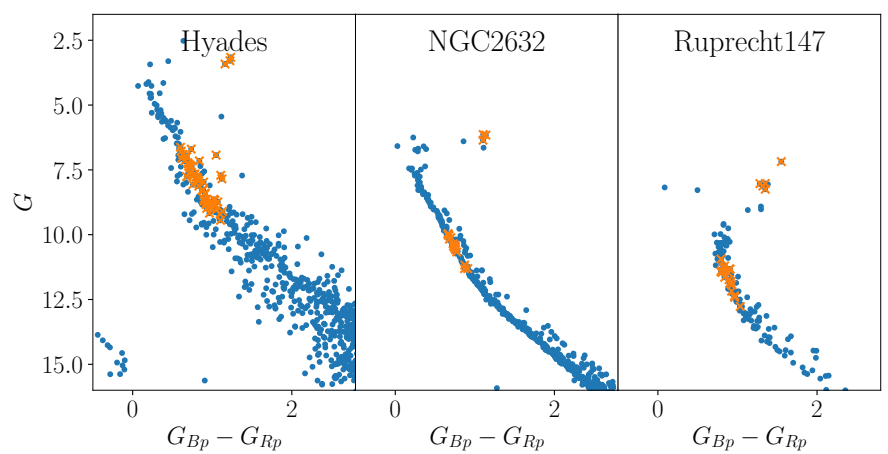

Fig. 1. Color-magnitude diagrams of the stars of the three clusters. In blue, we plotted the initial list of members from the studies indicated in the text. Target stars with retrieved spectra are plotted with orange crosses.

corresponded to the same star, we coadded them to reach a higher S/N. Several stars were also observed with different instruments, in this case, the spectra were treated independently for comparison purposes. We show in Fig. 1 a color-magnitude diagram of the cluster members, indicating the targets with spectra.

\section{Method}

We used the public spectroscopic software iSpec (BlancoCuaresma et al. 2014a; Blanco-Cuaresma 2019) to analyze the spectra. This is a Python code designed to perform operations on stellar spectra and to compute radial velocities, atmospheric parameters, and individual chemical abundances using different available atmospheric models and radiative transfer codes.

We employed the synthetic spectral synthesis method to compute atmospheric parameters and chemical abundances using the radiative transfer code SPECTRUM (Gray \& Corbally 1994), the MARCS ${ }^{7}$ atmospheric models (Gustafsson et al. 2008), and the solar abundances by Grevesse et al. (2007). We used the line list from the Gaia-ESO survey (Heiter et al. 2015a, 2019). The spectral fitting was done by comparing the observed fluxes weighted by their uncertainties with a synthetic spectrum for a set of spectral features. Atmospheric parameters and chemical abundances were varied in two separate steps until convergence was reached using a least-squares algorithm.

\section{Pipeline}

We adapted the pipeline used in Blanco-Cuaresma \& FraixBurnet (2018), which uses the general workflow described as follows. In a first preprocessing step, each spectrum is cut to a restricted common wavelength range $(480-680 \mathrm{~nm})$ and downgraded to a common resolution (45000) in order to be analyzed homogeneously. Heliocentric radial velocities are computed and the different spectra from the same star and instrument are coadded to reach a high $\mathrm{S} / \mathrm{N}$. The radial velocity of the coadded spectrum is determined from cross-correlation with a high $\mathrm{S} / \mathrm{N}$ solar spectrum from NARVAL. Strong telluric absorption or emission lines are identified and masked using a telluric line list. The spectrum is normalized to the continuum using quadratic splines, with nodes distributed along the spectrum at every $5 \mathrm{~nm}$. The continuum level is found using a median and maximum filter in order to account for the absorption lines.

\footnotetext{
7 http://marcs.astro.uu.se/
} 
Table 2. Selection of lines used to compute chemical abundances.

\begin{tabular}{lcccc}
\hline \hline Element & $\lambda_{\text {peak }}$ & $\log g f$ & EP & Stars \\
\hline Ca I & 534.9465 & -0.310 & 2.7090 & CD,WD \\
Ca I & 526.1704 & -0.579 & 2.5210 & WD,G \\
\hline
\end{tabular}

Notes. We indicate for each line: the element, wavelength $\left(\lambda_{\text {peak }}\right)$, atomic information (log $g f$ and excitation potential -EP-), and the type of stars. The full table is available at CDS.

The atmospheric parameters $T_{\mathrm{eff}}, \log g,[\mathrm{M} / \mathrm{H}]$, and $[\alpha / \mathrm{M}]$, as well as the microturbulence parameter $v_{\text {mic }}$ are inferred for each spectrum using spectral synthesis fitting, in a nondifferential way. We used the master line list by Blanco-Cuaresma (2019) and also the wings of $\mathrm{H} \alpha$ and $\mathrm{H} \beta$, as well as the Mg I b triplet lines.

As for the broadening effects, the projected equatorial rotational velocity $v \sin i$, the macroturbulence parameter, and the spectral resolution are degenerate and difficult to disentangle. We applied the strategy described in Blanco-Cuaresma (2019): We used a fixed value for $v \sin i$ of $1.6 \mathrm{~km} \mathrm{~s}^{-1}$, the macroturbulence was computed with the empirical relation used in the Gaia-ESO Survey (Bergemann \& Hill, priv. comm.), and only the spectral resolution was let free, accounting for all broadening effects.

Absolute chemical abundances of individual lines were measured using the atmospheric parameters fixed to the values resulting from the previous step. To derive chemical abundances, an additional cleaning of lines was done by systematically discarding discrepant lines of each element in most of the stars. This was done for three groups of stars according to evolutionary state: K giants $(\log g<3.5), \mathrm{G}$ dwarfs $\left(5000<T_{\text {eff }}<5900 \mathrm{~K}\right)$, and $\mathrm{F}$ dwarfs $\left(5900<T_{\text {eff }}<6400 \mathrm{~K}\right)^{8}$. For elements with few measured spectral lines, we used the flags included in the GaiaESO line list, indicating the reliability of the atomic data and their degree of blending. We tested that the obtained lines were consistent by using stars observed with different instruments. The final selection of lines is in Table 2.

In the final step, differential abundances were calculated line by line, by subtracting the abundance values of a chosen reference star (see Sect. 6). This procedure was restricted to those lines present in the reference star.

\section{Galactic velocities: Membership refinement}

We used radial velocities computed by iSpec to identify kinematic outliers in each cluster. Such stars could be nonmembers (or less reliable members), or spectroscopic binaries, which we want to remove from our sample to retrieve chemical abundances of member stars whose chemical pattern reflects the composition of the gas cloud, and not anomalies due to binary interaction. We find compatible radial velocities among different spectra of the same star. Several stars have significantly different radial velocities with respect to the rest of the cluster stars. This is expected because of the projection effects on the sky in these nearby objects, in particular, for the Hyades and NGC 2632.

We identified nine stars with large uncertainties in radial velocity $\left(>1.5 \mathrm{~km} \mathrm{~s}^{-1}\right)$, which tend to have large FWHM of the spectral features $\left(\gtrsim 30 \mathrm{~km} \mathrm{~s}^{-1}\right)$. We list them in the upper part of

\footnotetext{
8 These limits in temperature do not correspond to the exact definition of spectral types. However, the two groups are dominated by the $\mathrm{G}$ and $\mathrm{F}$ type stars, respectively. We use this nomenclature throughout the paper for simplicity.
}

Table 3. Stars with large $v_{\mathrm{r}}$ uncertainty (upper part), and identified outliers using total velocities (lower part).

\begin{tabular}{lcc}
\hline \hline Cluster & Star & Comments \\
\hline Hyades & 43789772861265792 & EB (V471 Tau) \\
Hyades & 144130516816579200 & Tidal tails \\
Hyades & 3312837025641272320 & Tidal tails \\
Hyades & 149313099234711680 & \\
Hyades & 3305871825637254912 & \\
Hyades & 3314212068010812032 & \\
Hyades & 3393284752392701312 & \\
NGC 2632 & 1918687411545919232 & Tidal tails \\
NGC 2632 & 661419259867455488 & \\
\hline Hyades & 2495442626804315392 & Tidal tails \\
Hyades & 3380479015342121600 & Tidal tails \\
Hyades & 145293181643038336 & SB ${ }^{(1)}$ \\
Rup 147 & 4087807180650392832 & \\
Rup 147 & 4087786874044570880 & \\
Rup 147 & 4184144534049662720 & Lit. discrepant $v_{\mathrm{r}}{ }^{(2)}$, SB? \\
\hline
\end{tabular}

Notes. We indicate the cluster and the Gaia DR2 source id. $\mathrm{SB}=$ spectroscopic binary.

References. ${ }^{(1)}$ White et al. (2007). ${ }^{(2)}$ Gaia Collaboration (2018b), Curtis et al. (2013).

Table 3 . These correspond to warm stars $\left(T_{\text {eff }} \gtrsim 6300 \mathrm{~K}\right)$, which possibly rotate more rapidly than solar-type stars (Nielsen et al. 2013). The uncertainties in the abundances of all these stars are significantly larger than for the rest of our sample. We exclude all of them for the analysis in the next subsections because it is not possible to retrieve reliable abundances with our employed procedure of differential analysis. In particular, we remark that Gaia DR2 43789772861265792 (V471 Tau) has a FWHM that is 100 times larger than the rest of the stars. This is one of the giant stars in the Hyades, which is a known eclipsing binary.

We used Gaia DR2 proper motions and positions, and the derived radial velocities to compute Galactic $6 \mathrm{D}$ coordinates $(X, Y, Z, U, V, W)$ using pygai ${ }^{9}$. For the stars of each of the three clusters, we applied a $3 \sigma$ rejection until a dispersion in each velocity coordinate of $<2.5 \mathrm{~km} \mathrm{~s}^{-1}$ was reached, corresponding to the typical dispersion expected for a cluster (Riedel et al. 2017). We identified three discrepant stars in the Hyades and three in Ruprecht 147. See the lower part of Table 3 for a summary of these stars. We removed them from the next subsections. Several of the outliers correspond to the preceding and trailing tidal tails of the Hyades, these are analyzed in Sect. 6.5. One of the Hyades outliers has been previously identified in the literature as a spectroscopic binary. From the outliers identified in Ruprecht 147, two do not have previous measurements in the literature. The other has two previous measures, which are discrepant among them and with our value. It is a possible spectroscopic binary.

The median and MAD of the cluster radial velocities that were determined with our set of high resolution spectra (excluding outliers from Galactic velocities) are $39 \pm 2 \mathrm{~km} \mathrm{~s}^{-1}$ for the Hyades (59 stars), $34.4 \pm 0.8 \mathrm{~km} \mathrm{~s}^{-1}$ for NGC 2632 (22 stars), and $41.4 \pm 0.5 \mathrm{~km} \mathrm{~s}^{-1}$ for Rup 147 (21 stars). These values are in good agreement with the mean radial velocities that were derived by Gaia Collaboration (2018a) and Soubiran et al. (2018) from Gaia DR2 data.

9 https://github.com/agabrown/PyGaia 

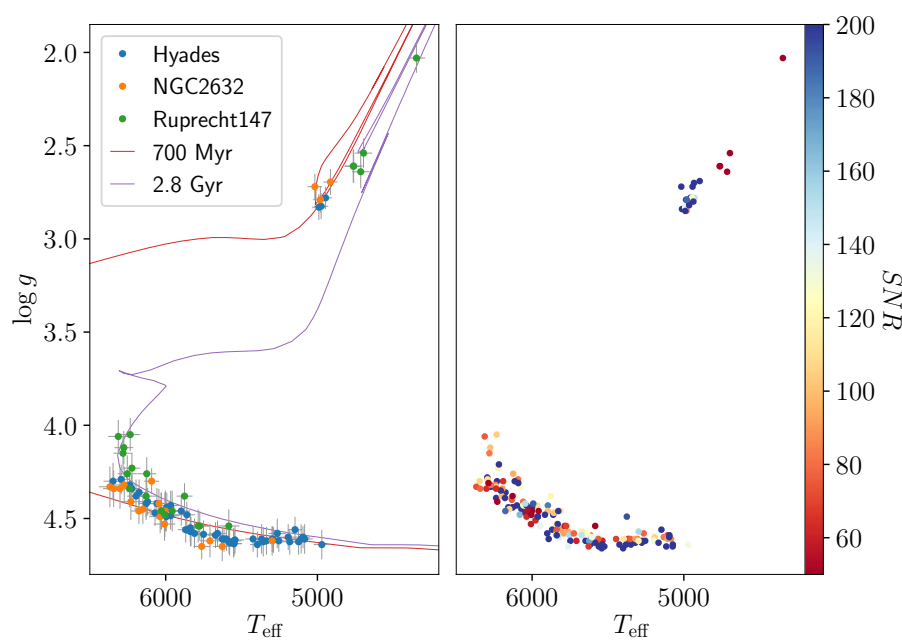

Fig. 2. HR diagram showing the $T_{\text {eff }}$ and $\log g$ values resulting from the analysis of the target stars. Left: stars are colored according to the cluster, using median values for the stars with spectra from different instruments. We overplotted two isochrones representative of the ages of the clusters. Right: all spectra colored by $\mathrm{S} / \mathrm{N}$.

\section{Results of the spectroscopic analysis}

\subsection{Atmospheric parameters}

We show in Fig. 2 the $T_{\text {eff }}$ and $\log g$ resulting from the analysis of the bona fide member stars. The uncertainties were computed by adding the quoted uncertainty delivered by iSpec to the mean dispersion obtained from the comparison of the GBS (see Table 5). We overplotted two isochrones ${ }^{10}$ that are representative of the ages of the Hyades and Praesepe (700 Myr) and Ruprecht 147 (2.8 Gyr). One can see three clear main sequences and red clumps corresponding to the three clusters. The locus of the main-sequence turn-off and of the giants in Ruprecht 147 differ slightly from the other two because of its older age. One giant star in Ruprecht 147 is brighter and cooler than its red clump (Gaia DR2 4183949198935967232). This star was identified as a red giant branch star by Carlberg (2014).

We used the sample of stars that were observed with more than one instrument to check the internal consistency of the atmospheric parameters. In total, 44 stars have several observations. In Fig. 3 we show, for each instrument, the difference in $T_{\text {eff }}$ and $\log g$ between the value obtained for the spectrum of that instrument and the value for the same star observed with other instruments. The median offsets in $T_{\text {eff }}$ and $\log g$ are lower than $22 \mathrm{~K}$ and $0.05 \mathrm{dex}$, respectively, and the dispersions (MAD) are of a similar level. Only for $T_{\text {eff }}$ in ESPaDOnS we obtain an offset that is significantly larger than the dispersion, but in this case, we have a very small number of stars.

\subsection{Chemical abundances}

We computed $\mathrm{LTE}^{11}$ chemical abundances as explained in Sect. 3 for 22 chemical species of all nucleosynthetic channels: Na I, Al I, Mg I, Si I, Ca I, Sc II, Ti I, Ti II, VI, Cr I, Mn I, Fe I, Fe II, Co I, Ni I, Cu I, Y II, Ba II, La II, Ce II, Nd II, and Eu II. We selected the lines that were computed for each element by systematically discarding discrepant lines, as explained in Sect. 3.

\footnotetext{
${ }_{10}$ PARSEC isochrones (Marigo et al. 2017), http://stev.oapd. inaf.it/cgi-bin/cmd

${ }^{11}$ Local Termodynamic Equilibrium.
}

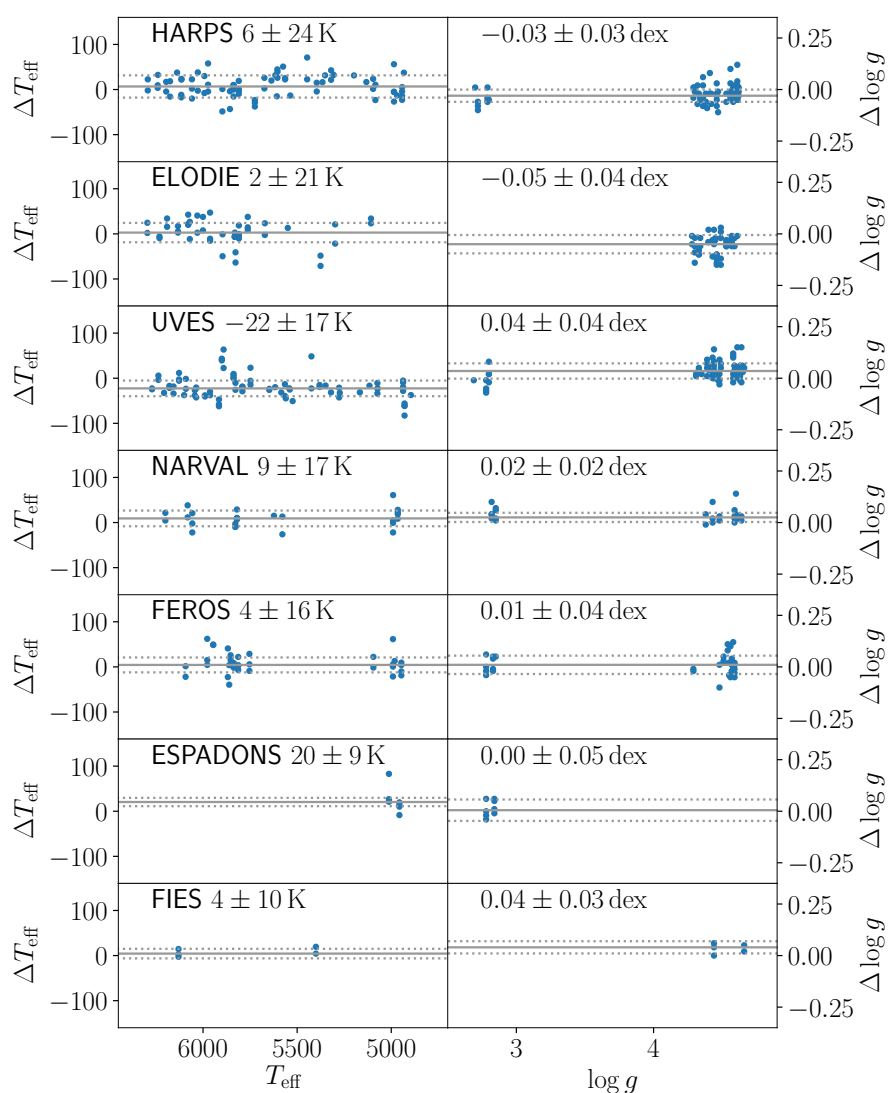

Fig. 3. Differences in $T_{\text {eff }}$ (left column) and $\log g$ (right column) for stars observed with different instruments. For each instrument we show the difference as: instrument - others, as a function of the value obtained for that instrument. We indicate the median and MAD difference in each panel.

Several elements could only be analyzed in certain spectral types ( $\mathrm{La}, \mathrm{Ce}$ ), according to the line selection made in Sect. 3.

From the absolute ${ }^{12}$ chemical abundances retrieved from iSpec, we computed bracket abundances with respect to the Sun $([\mathrm{X} / \mathrm{H}])$ by using the median solar abundance obtained with the nine available spectra from the Sun that were analyzed within the GBS (see next subsection). The results are plotted in Fig. 4 for the stars in each cluster and sorted by $T_{\text {eff }}$. Stars that were observed several times are represented by the mean value of the element abundance, and their uncertainty is computed as the squared sum of the standard deviation and the mean of the quoted errors. Abundances with large uncertainties $(>0.2$ dex) are rejected in the discussion and the plot; this considerably lowers the number of stars in heavy elements (Ce II, Nd II, and Eu II).

Several chemical species, such as $\mathrm{NaI}, \mathrm{Mg}$ I, VI, Mn I, and La II, show a significant gradient in abundance with effective temperature and surface gravity. These differences can be due to several effects. (1) Non-LTE effects are expected to be large in some of these elements, such as $\mathrm{Na}$ (Lind et al. 2011), up to $0.5 \mathrm{dex}$, and Mn (Bergemann et al. 2019) up to 0.4 dex. (2) It can also be due to a change in chemical abundances in the stellar atmosphere depending on the evolutionary stage. Several studies have found significant abundance variations among stars across different evolutionary phases in old OCs (such as M 67,

\footnotetext{
12 Absolute abundance is defined as $A_{\mathrm{X}}=\log \left(\frac{N_{\mathrm{X}}}{N_{\mathrm{H}}}\right)+12$ where $N_{\mathrm{X}}$ and $N_{\mathrm{H}}$ are the number of absorbers of the element atoms, and of hydrogen, respectively.
} 


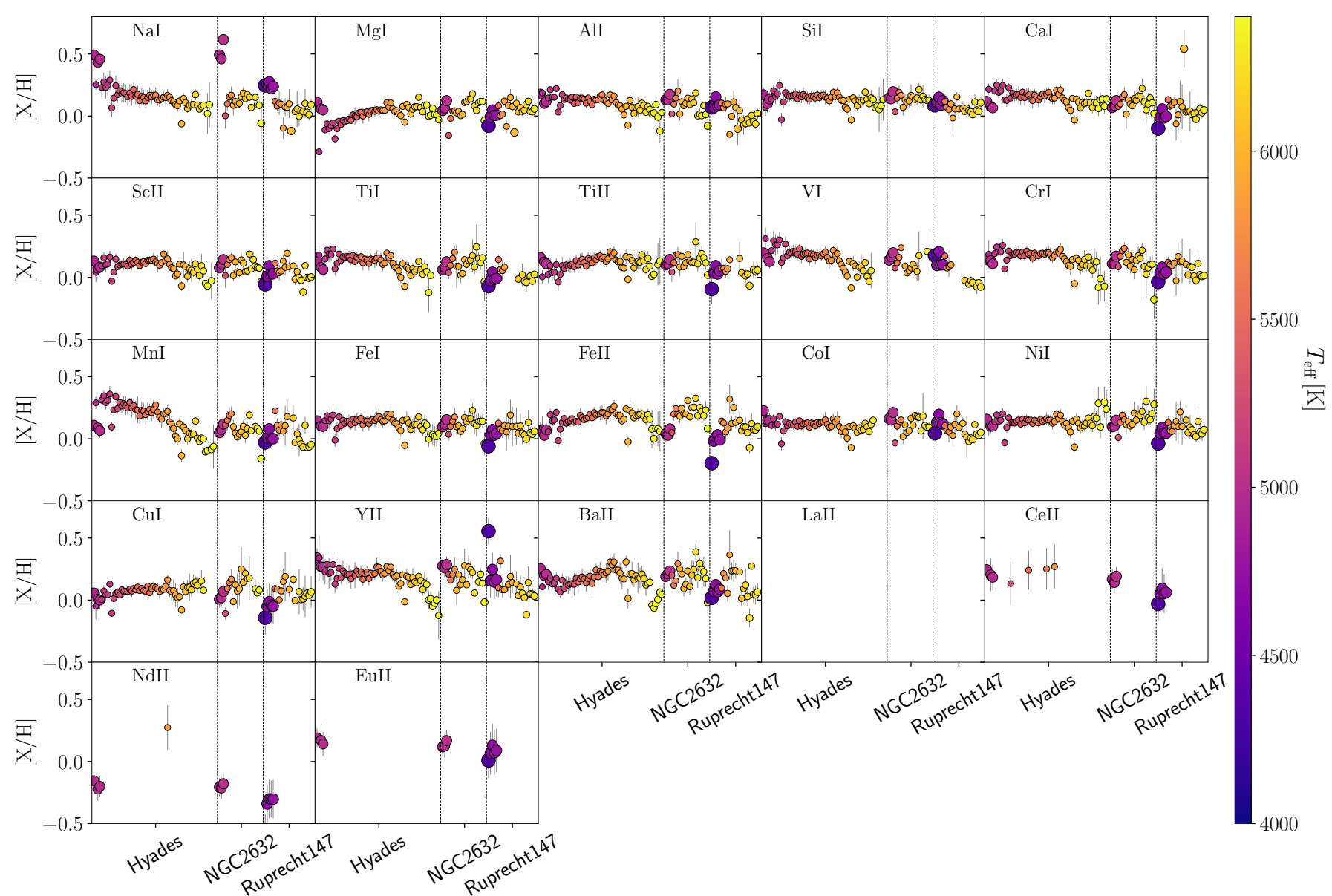

Fig. 4. Abundances $[\mathrm{X} / \mathrm{H}]$ of each chemical species computed for the stars in the analyzed OCs. The color codes the effective temperatures and the size represents the surface gravity (larger sizes correspond to giant stars). Vertical lines separate the stars of the three clusters.

see e.g., Souto et al. 2018), which have been attributed to the effects of diffusion. In general, diffusion causes surface abundances to decrease along the main sequence by up to $\sim 0.1$ dex in certain elements (Dotter et al. 2017). After the turnoff and up to the red giant branch, convection erases these effects, thus restoring the original abundances. The order of magnitude of diffusion is expected to be the largest in $\mathrm{Na}, \mathrm{Mg}, \mathrm{Al}$, and $\mathrm{Fe}$. We do see differences in $\mathrm{Na}$ and $\mathrm{Mg}$ (though for $\mathrm{Mg}$ the gradient is in the opposite direction), but we do not see a clear sign in $\mathrm{Al}$ and Fe. (3) This effect can also be due to the systematics introduced by the analysis, for example, due to unidentified blends that are stronger for a certain temperature of the star (see Jofré et al. 2019, for a review). Elements with few lines are probably more affected by this (e.g., this could be the case for $\mathrm{Mg}$ ). Also, we see a difference between the abundances of Fe I and Fe II, the latter probably has a dependence in $T_{\text {eff }}$ because of the fewer visible lines.

In conclusion, bracket abundances are affected by different systematic effects that are difficult to disentangle, and that depends on spectral type and age in different ways. It is difficult to reach a conclusion as to which effect plays a significant role in each element. As seen in Sect. 6, this can be solved by computing differential abundances.

Given the observed differences depending on the evolutionary state, and in order to give reference values of bracket abundances, we computed the median abundance and its MAD for different spectral types. In Table 4 we list the final bracket abundance values for F dwarfs, G dwarfs, and $\mathrm{K}$ giants of the three clusters. We remark that Fe II and Fe II are not in agreement in some cases, for instance, for F dwarfs and K giants in NGC 2632 differences are up to $0.07 \mathrm{dex}$. This is a direct consequence of the trend with temperature seen in Fe II and not in the neutral case. On the contrary, in general, the two states of Ti seem to agree more among them.

\subsection{Gaia FGK benchmark stars}

The GBS are a set of reference stars, which cover different regions of the HR diagram and a wide range in metallicity. For these stars, the effective temperature and surface gravity were determined independently from spectroscopy (Heiter et al. 2015b; Hawkins et al. 2016). Reference metallicities (Jofré et al. 2014; Hawkins et al. 2016) and abundances (Jofré et al. 2015) also exist. They are widely used in the community for the cross-calibration and validation of pipelines and spectroscopic analyses. A summary description of their latest atmospheric parameters can be found in Jofré et al. (2018).

We used the sample of the GBS high-quality spectra from the spectral library by Blanco-Cuaresma et al. (2014b) to test the atmospheric parameters resulting from our pipeline. We also queried the archives for more spectra of these stars. We selected a subset of the whole sample of GBS according to the parameter space covered by the cluster stars: those classified as FGK dwarfs (with $6500 \mathrm{~K}<T_{\text {eff }}<4900 \mathrm{~K}$ ), FGK giants, and excluding metal-poor stars $([\mathrm{M} / \mathrm{H}]<-1)$. With this selection, we obtained 184 spectra of 16 GBS. We processed these spectra using the same pipeline as the one used for the cluster stars. 
Table 4. Cluster average abundances (w.r.t. the Sun) and dispersions, weighted by the uncertainty.

\begin{tabular}{|c|c|c|c|c|c|c|c|c|}
\hline \multirow[b]{2}{*}[\mathrm{X}/\mathrm{H}]{} & \multicolumn{3}{|c|}{ Hyades } & \multicolumn{2}{|c|}{ NGC 2632} & \multicolumn{3}{|c|}{ Ruprecht 147} \\
\hline & F Dwarfs & G Dwarfs & K Giants & F Dwarfs & K Giants & F Dwarfs & G Dwarfs & K Giants \\
\hline $\mathrm{NaI}$ & $0.09 \pm 0.04$ & $0.16 \pm 0.04$ & $0.46 \pm 0.02$ & $0.10 \pm 0.05(15)$ & $0.49 \pm 0.06$ & $0.06 \pm 0.04$ & $0.10 \pm 0.01$ & $0.25 \pm 0.01$ \\
\hline $\operatorname{Mg} I_{1}$ & $0.05 \pm 0.04(23)$ & $0.00 \pm 0.06$ & $0.06 \pm 0.03$ & $0.12 \pm 0.05(15)$ & $0.06 \pm 0.03$ & $0.07 \pm 0.04$ & $0.04 \pm 0.04$ & $0.01 \pm 0.03$ \\
\hline $\mathrm{AlI}$ & $0.06 \pm 0.03$ & $0.14 \pm 0.02$ & $0.12 \pm 0.03$ & $0.08 \pm 0.06(15)$ & $0.13 \pm 0.02$ & $-0.03 \pm 0.06(15)$ & $0.08 \pm 0.03$ & $0.08 \pm 0.02(5)$ \\
\hline Si I & $0.11 \pm 0.04$ & $0.15 \pm 0.03$ & $0.14 \pm 0.02(3)$ & $0.13 \pm 0.03(15)$ & $0.14 \pm 0.02(3)$ & $0.03 \pm 0.03(14)$ & $0.08 \pm 0.02$ & $0.11 \pm 0.02$ \\
\hline $\mathrm{Ca} \mathrm{I}$ & $0.11 \pm 0.04$ & $0.17 \pm 0.03(36)$ & $0.08 \pm 0.02(3)$ & $0.12 \pm 0.05(15)$ & $0.09 \pm 0.02(3)$ & $0.03 \pm 0.03(14)$ & $0.10 \pm 0.02$ & $-0.00 \pm 0.04$ \\
\hline Sc II & $0.03 \pm 0.06(23)$ & $0.12 \pm 0.03$ & $0.10 \pm 0.02$ & $0.06 \pm 0.03(15)$ & $0.11 \pm 0.02(3)$ & $0.00 \pm 0.05$ & $0.05 \pm 0.03$ & $0.01 \pm 0.03$ \\
\hline Ti I & $0.06 \pm 0.03$ & $0.15 \pm 0.02$ & $0.08 \pm 0.03$ & $0.11 \pm 0.04$ & $0.07 \pm 0.03$ & $-0.01 \pm 0.01$ & $0.06 \pm 0.03$ & $-0.01 \pm 0.03$ \\
\hline Ti II & $0.09 \pm 0.04$ & $0.13 \pm 0.03$ & $0.09 \pm 0.01$ & $0.15 \pm 0.05$ & $0.05 \pm 0.02$ & $0.01 \pm 0.01$ & $0.07 \pm 0.02$ & $0.00 \pm 0.03$ \\
\hline V I & $0.05 \pm 0.05$ & $0.19 \pm 0.03$ & $0.16 \pm 0.03(3)$ & $0.07 \pm 0.04(11)$ & $0.16 \pm 0.02$ & $-0.04 \pm 0.02$ & $0.09 \pm 0.02$ & $0.07 \pm 0.03$ \\
\hline CrI & $0.10 \pm 0.04(20)$ & $0.19 \pm 0.02$ & $0.13 \pm 0.02$ & $0.12 \pm 0.04(13)$ & $0.15 \pm 0.02(2)$ & $0.03 \pm 0.04(12)$ & $0.12 \pm 0.01$ & $0.04 \pm 0.03$ \\
\hline Mn I & $0.04 \pm 0.06(21)$ & $0.23 \pm 0.05(36)$ & $0.05 \pm 0.02(3)$ & $0.09 \pm 0.04(15)$ & $0.04 \pm 0.03$ & $-0.01 \pm 0.05$ & $0.07 \pm 0.03$ & $-0.02 \pm 0.03$ \\
\hline Fe I & $0.10 \pm 0.05(21)$ & $0.15 \pm 0.02$ & $0.10 \pm 0.02$ & $0.14 \pm 0.04$ & $0.10 \pm 0.03$ & $0.04 \pm 0.05$ & $0.11 \pm 0.02$ & $0.02 \pm 0.03$ \\
\hline Fe II & $0.15 \pm 0.07(23)$ & $0.17 \pm 0.03$ & $0.06 \pm 0.02$ & $0.20 \pm 0.04(15)$ & $0.03 \pm 0.02$ & $0.09 \pm 0.05$ & $0.11 \pm 0.03$ & $-0.02 \pm 0.05(5)$ \\
\hline CoI & $0.08 \pm 0.03(18)$ & $0.13 \pm 0.02$ & $0.15 \pm 0.03$ & $0.09 \pm 0.06$ & $0.15 \pm 0.03$ & $0.04 \pm 0.05$ & $0.07 \pm 0.03$ & $0.09 \pm 0.02(5)$ \\
\hline $\mathrm{Ni}$ & $0.11 \pm 0.04$ & $0.14 \pm 0.02$ & $0.10 \pm 0.03$ & $0.15 \pm 0.03$ & $0.10 \pm 0.02$ & $0.07 \pm 0.02(11)$ & $0.08 \pm 0.03$ & $0.05 \pm 0.03$ \\
\hline $\mathrm{Cu} \mathrm{I}$ & $0.08 \pm 0.06(22)$ & $0.08 \pm 0.03$ & $0.01 \pm 0.02$ & $0.13 \pm 0.07(15)$ & $0.02 \pm 0.03$ & $0.07 \pm 0.05(12)$ & $0.08 \pm 0.03$ & $-0.05 \pm 0.03(5)$ \\
\hline Y II & $0.14 \pm 0.08$ & $0.20 \pm 0.03$ & $0.37 \pm 0.02$ & $0.16 \pm 0.05(15)$ & $0.41 \pm 0.04$ & $0.04 \pm 0.07(13)$ & $0.07 \pm 0.09$ & $0.25 \pm 0.04$ \\
\hline Ba II & $0.17 \pm 0.08$ & $0.17 \pm 0.04$ & $0.21 \pm 0.02$ & $0.21 \pm 0.07$ & $0.21 \pm 0.01$ & $0.11 \pm 0.11$ & $0.14 \pm 0.06$ & $0.07 \pm 0.03(5)$ \\
\hline La II & & $0.17 \pm 0.06$ & $-0.05 \pm 0.02$ & & $-0.05 \pm 0.02$ & - & $0.02 \pm 0.05$ & $-0.09 \pm 0.04$ \\
\hline Ce II & & $0.19 \pm 0.05(20)$ & $0.20 \pm 0.03$ & & $0.19 \pm 0.03$ & & $0.26 \pm 0.00(1)$ & $0.09 \pm 0.04(5)$ \\
\hline Nd II & $-0.16 \pm 0.06(13)$ & $0.14 \pm 0.06$ & $-0.20 \pm 0.02$ & $-0.14 \pm 0.03(12)$ & $-0.21 \pm 0.02$ & $-0.11 \pm 0.17(13)$ & $0.06 \pm 0.03$ & $-0.27 \pm 0.03$ \\
\hline Eu II & & & $0.17 \pm 0.02(3)$ & $0.07(1)$ & $0.12 \pm 0.02$ & & & $0.07 \pm 0.03$ \\
\hline
\end{tabular}

Notes. For each cluster we give a value of: F dwarfs, G dwarfs, and K giants. The number of stars in each group and element is indicated in parenthesis.

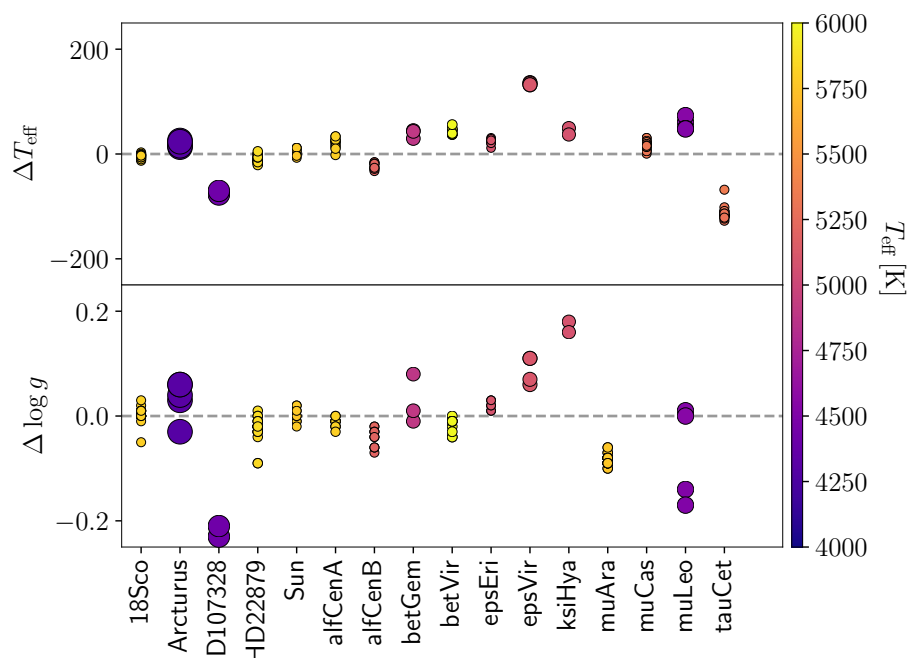

Fig. 5. Differences (here - reference values from Jofré et al. 2018) in $T_{\text {eff }}$ and $\log g$ for the selection of the GBS. The colors correspond to the temperature and the sizes are scaled with the inverse of the surface gravity (larger symbols mean giant stars). Vertically aligned symbols correspond to different spectra of the same star.

In Fig. 5 we plotted the obtained $T_{\text {eff }}$ and $\log g$ compared with the reference (Heiter et al. 2015b; Jofré et al. 2018) for each spectrum. We obtain a good agreement, with differences below $100 \mathrm{~K}$ and 0.1 dex in $T_{\text {eff }}$ and $\log g$, respectively, with, however, larger differences for the giants HD 107328, $\epsilon$ Vir, and $\xi$ Hya, especially for the surface gravity. Remarkably seen in Fig. 5, for a given star, the analysis of the spectra even from different instruments return values in very good internal agreement up to $15 \mathrm{~K}$ in $T_{\text {eff }}$ and 0.02 dex in $\log g$, except for two of the spectra of $\mu$ Leo. A summary of the mean differences by spectral type (the label "Group" is indicated in the GBS reference table) is found
Table 5. Comparison of the results of $T_{\text {eff }}$ and $\log g$ for the GBS with respect to the reference ones (see text).

\begin{tabular}{lcccc}
\hline \hline $\begin{array}{l}\text { Spectral } \\
\text { type }\end{array}$ & $\begin{array}{c}\text { Num } \\
\text { stars }\end{array}$ & $\begin{array}{c}\text { Num } \\
\text { spectra }\end{array}$ & $\begin{array}{c}\Delta T_{\text {eff }} \\
(\mathrm{K})\end{array}$ & $\begin{array}{c}\Delta \log g \\
(\mathrm{dex})\end{array}$ \\
\hline FGK giants & 6 & 20 & $46 \pm 57$ & $0.01 \pm 0.11$ \\
G dwarfs & 9 & 155 & $-17 \pm 49$ & $-0.03 \pm 0.03$ \\
K dwarfs & 1 & 9 & $24 \pm 5$ & $0.020 \pm 0.008$ \\
\hline All & 16 & 184 & $-7 \pm 53$ & $-0.02 \pm 0.06$ \\
\hline
\end{tabular}

Notes. Mean differences \pm standard deviations are listed, together with the number of stars and spectra for each spectral type.

in Table 5 where it is clear that we do not obtain any significant offset for a given spectral type.

The abundance values of the different spectra of the analyzed stars are plotted in Fig. B.1 for each analyzed element. The values of the median abundances per star and their uncertainties are listed in Tables A.1 and A.2. For most of the elements, the abundance dispersion (MAD) per star is small, on the order of 0.01-0.02 dex, reflecting the good agreement between lines of an element in the same star. The cases of $\mathrm{Eu}$ and $\mathrm{Ce}$ are the ones giving larger MAD, which is sometimes larger than 0.05 dex. This is possibly because their abundances are retrieved from the fit of very few weak lines in the spectra.

We did an external comparison by using the reference values from Jofré et al. (2015). This study provides reference abundances of iron-peak and $\alpha$ elements for the whole sample of the GBS. The mean values and standard deviations of the differences (this work - reference) per element are indicated in Table 6. All the differences, which are always lower than $0.05 \mathrm{dex}$, are consistent with the obtained dispersions and the quoted uncertainties. An exhaustive comparison star by star is plotted in Fig. B.2. 
Table 6. Mean (weighted by the uncertainty) element abundance difference obtained comparing this work with respect to the reference values of the GBS (Jofré et al. 2015).

\begin{tabular}{lc}
\hline \hline Element & Mean difference \\
\hline$\Delta[\mathrm{Fe} / \mathrm{H}]$ & $-0.04 \pm 0.08$ \\
$\Delta[\mathrm{Ca} / \mathrm{H}]$ & $-0.03 \pm 0.08$ \\
$\Delta[\mathrm{Co} / \mathrm{H}]$ & $0.01 \pm 0.08$ \\
$\Delta[\mathrm{Cr} / \mathrm{H}]$ & $-0.01 \pm 0.07$ \\
$\Delta[\mathrm{Mg} / \mathrm{H}]$ & $-0.05 \pm 0.07$ \\
$\Delta[\mathrm{Mn} / \mathrm{H}]$ & $0.02 \pm 0.08$ \\
$\Delta[\mathrm{Ni} / \mathrm{H}]$ & $-0.02 \pm 0.08$ \\
$\Delta[\mathrm{Sc} / \mathrm{H}]$ & $-0.04 \pm 0.08$ \\
$\Delta[\mathrm{Si} / \mathrm{H}]$ & $-0.03 \pm 0.07$ \\
$\Delta[\mathrm{Ti} / \mathrm{H}]$ & $-0.01 \pm 0.08$ \\
$\Delta[\mathrm{V} / \mathrm{H}]$ & $0.01 \pm 0.08$ \\
\hline
\end{tabular}

\section{Differential chemical abundances}

As a final step, we computed strictly line-by-line differential abundances in our pipeline as explained in Sect. 3. Differential abundances have been computed in several previous works for solar twins or solar analogs by using the Sun as a reference star (e.g., Meléndez et al. 2009; Tucci Maia et al. 2016; Liu et al. 2016a). This type of analysis provides high-precision abundances, erasing most of the effects that blur typical chemical abundance procedures, such as unaccounted blends, the effects of stellar evolution, and poor atomic line characterization. This technique has also been applied to other stellar types by selecting a reference star that is as close as possible to the analyzed stars in terms of stellar parameters (Reggiani et al. 2017; Hawkins et al. 2016; Jofré et al. 2015). In this case, the results tell us how much the abundances of the analyzed stars differ from those of the reference star, which is no longer necessarily the Sun. This is a good strategy to perform chemical tagging experiments.

In the case of the stars in the OCs analyzed here, we have a large spread in atmospheric parameters, and so performing a differential analysis is challenging. Therefore, we developed a strategy to perform a differential analysis not only for solar twins but by using stars at any evolutionary stage. We made eight groups of stars that differ among them by less than $\sim 200 \mathrm{~K}$, and $\sim 0.3 \mathrm{dex}$ in $T_{\text {eff }}$ and $\log g$, respectively, which are to be analyzed together as twins. For the upper main sequence and the giants, the limits in $\log g$ and $T_{\text {eff }}$ were relaxed to 0.45 dex in order to include, in the differential analysis, the warmest stars in the upper main sequence with the rest of the stars of the same temperature and the giants of Ruprecht 147 together with the giants from the other two clusters. The groups have between four and 16 stars in the three OCs and follow the main sequence and the giants. One star in each group was selected to be used as the reference to compute the differential abundances. See Fig. 6. Among the member stars in the three OCs, we were able to group 92 stars, leaving out those that fall out of the group limits in the $T_{\mathrm{eff}}-\log g$ plane.

The resulting abundance value of the element $X$, designated as $\delta X$, was computed as the mean of the abundance difference with respect to the reference for each line:

$\delta X=\frac{1}{N_{\text {lines }}} \sum_{i=1}^{N_{\text {lines }}}\left(A_{X_{i}}-A_{X_{i}, \mathrm{REF}}\right)$.

The reference stars selected for each group are different from each other. This could have consequences if one of the
Table 7. Reference stars used to compute differential abundances in each defined group.

\begin{tabular}{|c|c|c|c|c|}
\hline Star & $T_{\text {eff }}(\mathrm{K})$ & $\log g(\operatorname{dex})$ & {$[\mathrm{Fe} / \mathrm{H}](\mathrm{dex})$} & $N$ \\
\hline $\begin{array}{l}\text { Gaia DR2 } 3300934223858467072 \\
\text { HIP } 19796\end{array}$ & $6286 \pm 17$ & $4.30 \pm 0.04$ & $0.13 \pm 0.06$ & 3 \\
\hline $\begin{array}{l}\text { Gaia DR2 } 48203487411427456 \\
\text { HIP } 20237\end{array}$ & $6126 \pm 19$ & $4.41 \pm 0.04$ & 0.11 & 3 \\
\hline $\begin{array}{l}\text { Gaia DR2 } 3314109916508904064 \\
\text { HIP } 20899\end{array}$ & 5957 & $4.49 \pm 0.03$ & 0.12 & 4 \\
\hline $\begin{array}{l}\text { Gaia DR2 } 3313689422030650496 \\
\text { HIP } 20741\end{array}$ & $5834 \pm 19$ & $4.57 \pm 0.04$ & $0.17 \pm 0.05$ & 5 \\
\hline $\begin{array}{l}\text { Gaia DR2 } 144171233106399104 \\
\text { HIP } 21099\end{array}$ & $5582 \pm 23$ & $4.63 \pm 0.02$ & $0.14 \pm 0.04$ & 3 \\
\hline $\begin{array}{l}\text { Gaia DR2 } 3406823245223942528 \\
\text { HIP } 22380\end{array}$ & $5351 \pm 20$ & $4.61 \pm 0.04$ & $0.14 \pm 0.06$ & 1 \\
\hline $\begin{array}{l}\text { Gaia DR2 } 64266768177592448 \\
\text { HIP } 16908\end{array}$ & $5107 \pm$ & $4.61 \pm 0.03$ & $0.16 \pm 0.05$ & 1 \\
\hline $\begin{array}{l}\text { Gaia DR2 } 3312052249216467328 \\
\text { HIP } 20205\end{array}$ & $4975 \pm 12$ & $2.83 \pm 0.03$ & $0.10 \pm 0.05$ & 2 \\
\hline
\end{tabular}

Notes. We indicate the Gaia DR2 source ID, Hipparcos ID, the computed atmospheric parameters, iron bracket abundance, and the number of analyzed spectra. For stars with more than one spectra, we indicate the mean values and standard deviations of all determinations.

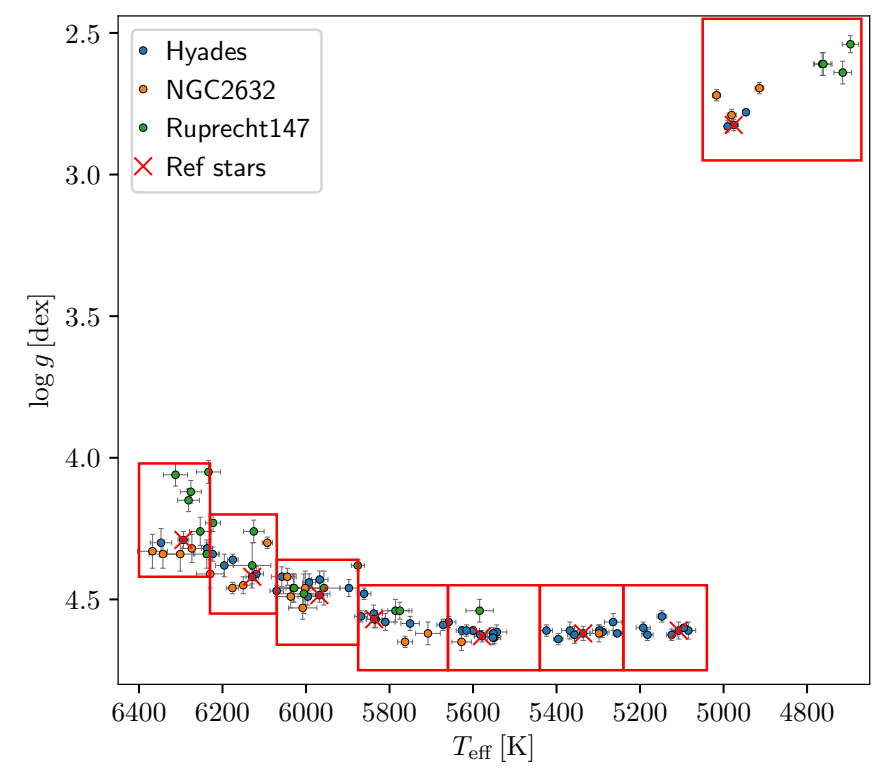

Fig. 6. HR diagram of the grouped stars in the three clusters. Colors represent each cluster as in Fig. 2. Red boxes indicate the $T_{\text {eff }}$ and $\log g$ limits of the groups used for the differential analysis. Red crosses mark the chosen reference stars.

chosen stars has a chemical peculiarity. In this case, the abundance scale for that group would be different from the others. For our experiment, the reference stars were chosen to be stars from the Hyades cluster that fall approximately in the middle of the $T_{\text {eff }}$ and $[\mathrm{Fe} / \mathrm{H}]$ range of each group. We have checked for previous information about these stars to be sure they have not been identified as high rotators or peculiar stars. We list the chosen reference stars with their atmospheric parameters in Table 7.

The resulting differential abundances for all chemical species are plotted for the three OCs in Fig. 7 as a function of $T_{\text {eff. }}$. We discarded the two stars in the tidal tails analyzed in Sect. 6.5 for not being members according to their chemical abundances. In this figure, the dependence with temperature has been erased for most of the elements, compared with the equivalent figure using bracket abundances (Fig. 4). 


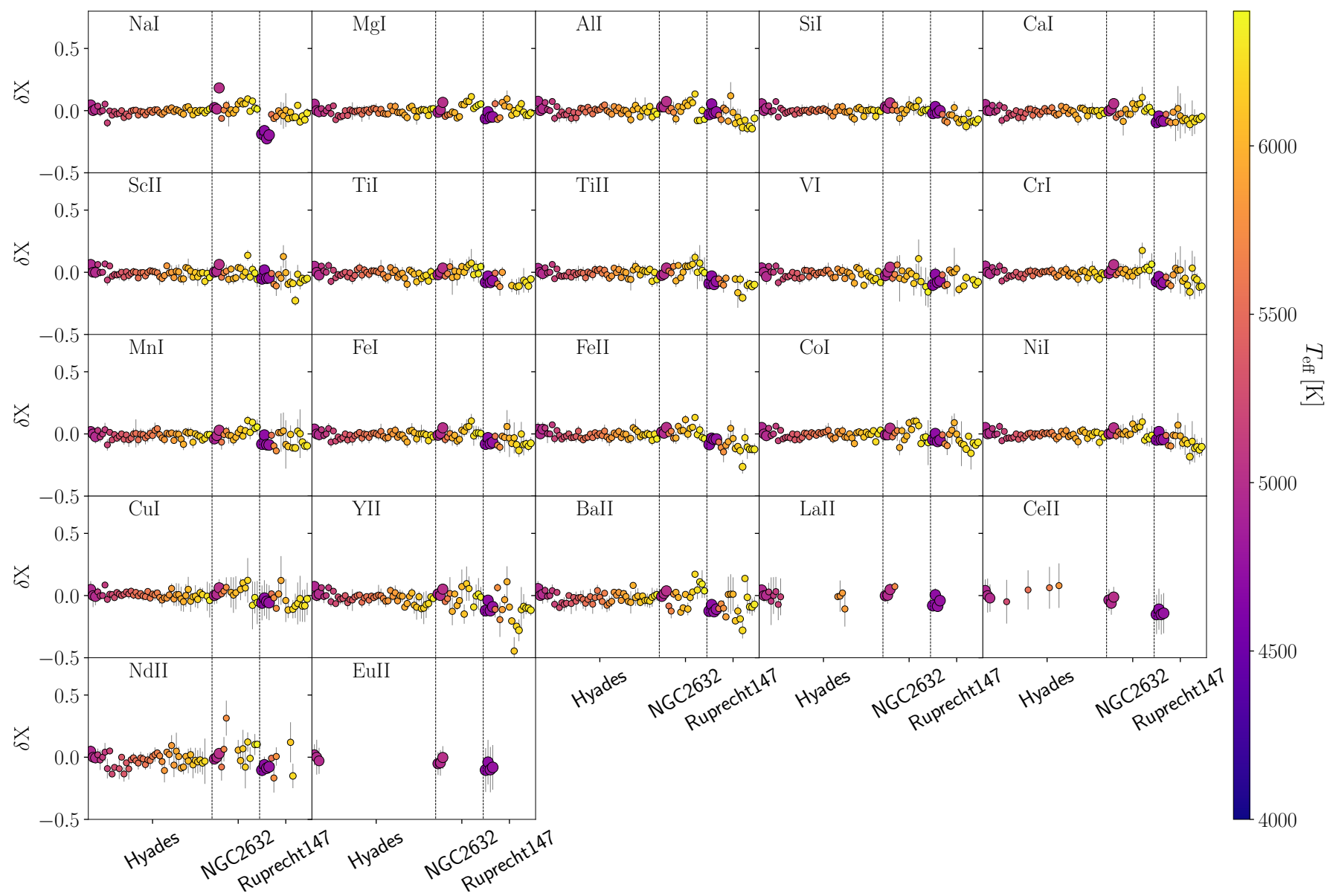

Fig. 7. Differential abundances as a function of effective temperature computed for the analyzed OCs. Colors and sizes are the same as in Fig. 4.

\subsection{Precision in differential abundances}

To assess our precision, we independently analyzed nine spectra of the same star, which were obtained with five different instruments. The chosen star is a dwarf from the Hyades, Gaia DR2 3313689422030650496. It was selected because it has spectra with different $\mathrm{S} / \mathrm{N}$ values, which are representative of the range that we have for the whole sample.

The resulting atmospheric parameters give a mean $T_{\text {eff }}$ of $5847 \mathrm{~K}$ (standard deviation of $11 \mathrm{~K}$, and a mean uncertainty of $25 \mathrm{~K}$ ), and the mean $\log g$ is $4.58 \mathrm{dex}$ (standard deviation of $0.02 \mathrm{dex}$, and mean of the quoted uncertainty of $0.03 \mathrm{dex}$ ). For the chemical abundance computation, we used the FEROS spectrum as a reference, and we computed line-by-line differential abundances using the same analysis pipeline for the bulk of the OC stars. In Fig. 8 we plot the resulting $T_{\text {eff }}, \log g$, and abundances of six representative species for all the spectra of this star. In Table 8 we list the dispersions in differential abundances found for all elements and the mean of the quoted uncertainties.

We do not obtain any clear systematics between instruments in the atmospheric parameters or the differential abundances. In general, the dispersions in abundances are on the order of $0.01-0.02$ dex. The mean quoted uncertainties tend to be larger $(0.03 \mathrm{dex})$ compared with the dispersions, which indicates that we might have slightly overestimated the uncertainties. The largest variations are seen for spectra that have $\mathrm{S} / \mathrm{Ns}$ at the lower end and for elements that present intrinsic difficulties to our pipeline for measuring abundances because of a few or weak lines (e.g., Y).

\subsection{Systematic uncertainties due to errors in atmospheric parameters}

We quantified the errors due to uncertainties in the stellar parameters in the same way as Jofré et al. (2015). We computed the differential abundances by using the same process as explained above, but by changing the value of the stellar parameters according to their uncertainties. The procedure was repeated eight times, by adding and subtracting the error in each stellar parameter: $T_{\text {eff }}, \log g, v_{\text {mic }}$, and $[\mathrm{Fe} / \mathrm{H}]$. For each parameter, the difference between the two values of the resulting abundance (adding and subtracting) is considered as the uncertainty: $\Delta T_{\text {eff }}$, $\Delta \log g, \Delta v_{\text {mic }}$, and $\Delta[\mathrm{Fe} / \mathrm{H}]$. Then we consider the total uncertainty would be

$\Delta=\sqrt{\Delta T_{\mathrm{eff}}^{2}+\Delta \log g^{2}+\Delta v_{\mathrm{mic}}^{2}+\Delta[\mathrm{Fe} / \mathrm{H}]^{2}}$

if the four parameters are statistically independent, which is not the case. A full covariance matrix should be computed, and then the total uncertainty would be smaller. However, this is a simpler and more conservative way of taking these uncertainties into account. See a more extended discussion in Jofré et al. (2019).

We have carried out this procedure for two representative stars that were observed with different instruments, a dwarf and a giant. The results for four chemical species are plotted in Fig. 9. Blue represents the mean value of the abundances that resulted to change the atmospheric parameters by their uncertainties, and the error bar represents the $\Delta$ from Eq. (2). For all chemical species, the quoted uncertainties in the differential abundances are larger than the change in the abundance due to atmospheric 


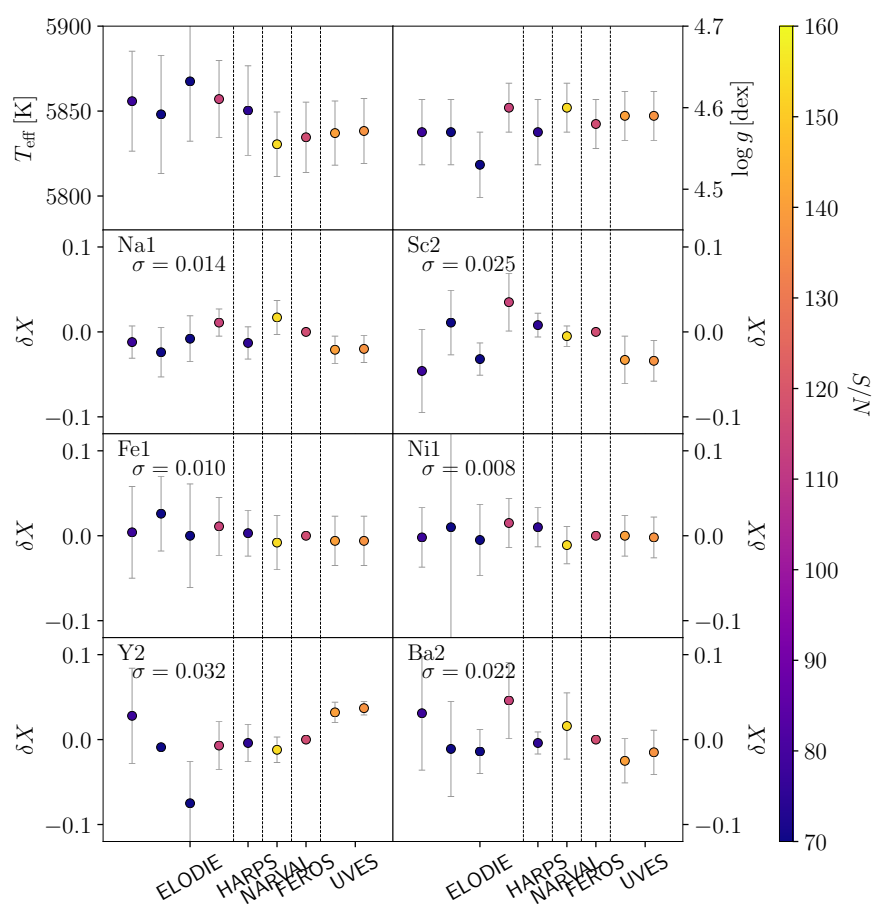

Fig. 8. First row: atmospheric parameters $\left(T_{\text {eff }}\right.$ and $\left.\log g\right)$ retrieved from the analysis of the different spectra of the star Gaia DR2 3313689422030650496. Last three rows: differential abundances of several chemical species for all spectra. The color code represents the $\mathrm{S} / \mathrm{N}$ of the spectra.

parameters. This shows that somehow these uncertainties are already representative of the effect of the uncertainties due to the atmospheric parameters.

\subsection{On the homogeneity of OCs}

The level of homogeneity of OCs has been investigated in several recent studies, using differential analysis of solar twins. Liu et al. (2016a, hereafter L16) studied 16 stars in the Hyades, Liu et al. (2016b) studied two stars in M 67, and Spina et al. (2018) analyzed five stars in the Pleiades. In the most extensive study, L16 computed abundances of 19 chemical species using high $\mathrm{S} / \mathrm{N}$ spectra of solar-type stars $\left(T_{\mathrm{eff}} \sim 5600-6300 \mathrm{~K}, \log g \sim\right.$ 4.47-4.58). They find total abundance variations of around 0.1 dex, and an abundance scatter from 0.020 to 0.045 dex (depending on the chemical species), in general, larger than the quoted uncertainties by a factor of $\sim 1.5$. Moreover, they find statistically significant correlations among the abundances of pairs of elements. They interpreted this as a signature of a genuine abundance scatter in the Hyades since it would mean that there is a difference in the overall metallicity between different stars. Otherwise, if differences only appeared for certain elements or for certain stars (i.e., scatter plot without correlations), this could either be caused by random errors or by certain processes in the stellar atmospheres depending on the stellar parameters.

\subsubsection{Observed scatter and correlations in abundances}

In this section, we investigate the homogeneity of the analyzed OCs. For the case of the Hyades, we have a larger sample than L16, 51 bonafide member stars for which we could compute differential abundances. We cover a range in $T_{\text {eff }}$ of 4900-6300 K, and in $\log g$ of 2.8-4.65 dex. For NGC 2632 and Ruprecht 147, we obtain differential abundances of 19 and 20 members, respectively.

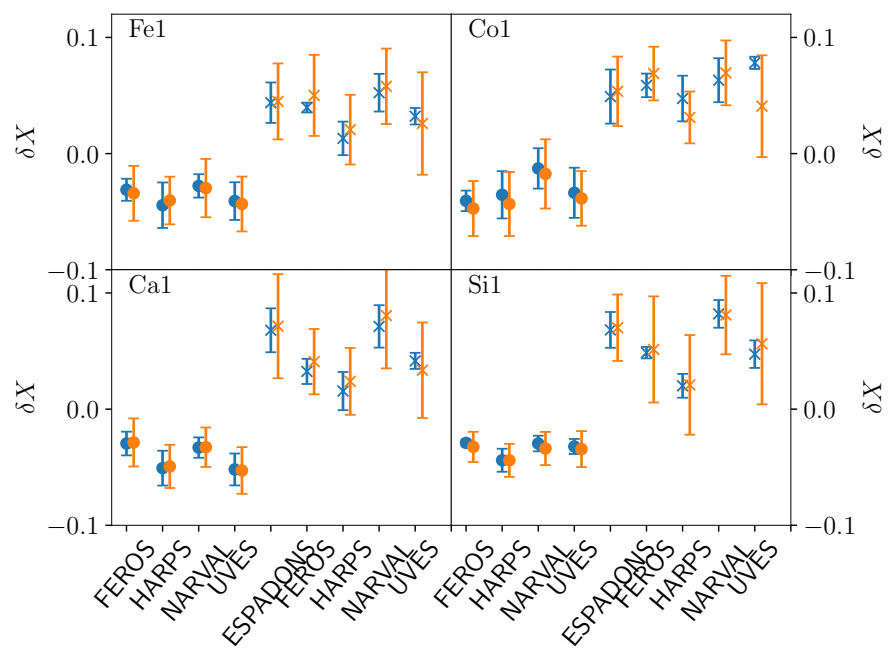

Fig. 9. Differential abundance results for different spectra of two stars, a dwarf (filled circles) and a giant (crosses). The values and uncertainties using the atmospheric parameters retrieved in Sect. 5.1 are in orange, and the values derived with the uncertainties in stellar parameters are in blue (see text).

Table 8. Dispersions and mean quoted uncertainties of the differential abundances found with the different spectra of the dwarf star Gaia DR2 3313689422030650496.

\begin{tabular}{lcc}
\hline \hline Element & $\begin{array}{c}\text { Abundance } \\
\text { dispersion }\end{array}$ & $\begin{array}{c}\text { Mean } \\
\text { uncertainty }\end{array}$ \\
\hline $\mathrm{Na}$ I & 0.016 & 0.019 \\
$\mathrm{Mg}$ I & 0.015 & 0.007 \\
$\mathrm{Al} \mathrm{I}$ & 0.015 & 0.010 \\
$\mathrm{Si} \mathrm{I}$ & 0.008 & 0.027 \\
$\mathrm{Ca}$ I & 0.013 & 0.025 \\
$\mathrm{Sc} \mathrm{II}$ & 0.023 & 0.018 \\
$\mathrm{Ti}$ I & 0.017 & 0.028 \\
$\mathrm{Ti}$ II & 0.018 & 0.034 \\
$\mathrm{~V} \mathrm{I}$ & 0.015 & 0.041 \\
$\mathrm{Cr}$ I & 0.009 & 0.039 \\
$\mathrm{Mn}$ I & 0.025 & 0.020 \\
$\mathrm{Fe}$ I & 0.011 & 0.033 \\
$\mathrm{Fe}$ II & 0.022 & 0.049 \\
$\mathrm{Co}$ I & 0.013 & 0.032 \\
$\mathrm{Ni}$ I & 0.007 & 0.037 \\
$\mathrm{Cu}$ I & 0.026 & 0.008 \\
Y II & 0.032 & 0.021 \\
$\mathrm{Ba}$ II & 0.023 & 0.029 \\
$\mathrm{Ce}$ II & 0.057 & - \\
Eu II & 0.076 & - \\
\hline
\end{tabular}

Notes. For elements without an uncertainty, the abundance comes from a single line. No La II and Nd II abundances are computed for this star.

In Table 9 we show the dispersion and amplitude of the differential abundances as well as the mean of the quoted uncertainties for each chemical species and each of the three clusters. The amplitudes of all elements are around 0.15 dex for the Hyades and 0.2 dex in the other two clusters. The abundance dispersions for the Hyades are typically on the order of 0.02-0.03 dex. In general, these are not larger than the quoted uncertainties, however, they are larger than the dispersions among the results of different spectra of the same star found in Sect. 6.1 (lower 
L. Casamiquela et al.: Differential abundances of nearby Open Clusters and their tidal tails

Table 9. Dispersion $(\sigma X)$ and full amplitude range of the abundances $\left(A_{X}\right.$, i.e., difference between highest and lowest value) and mean of the quoted uncertainties for each cluster (left) and comparison with L16 results for the elements in common (right).

\begin{tabular}{|c|c|c|c|c|c|c|c|c|c|}
\hline \multirow[t]{2}{*}{$\overline{\overline{\text { Cluster }}}$} & \multicolumn{3}{|c|}{ Hyades } & \multicolumn{3}{|c|}{ NGC 2632} & \multicolumn{3}{|c|}{ Ruprecht 147} \\
\hline & $\sigma X$ & $A_{X}$ & Unc. & $\sigma X$ & $A_{X}$ & Unc. & $\sigma X$ & $A_{X}$ & Unc. \\
\hline $\mathrm{Na} I$ & 0.025 & 0.151 & 0.021 & 0.052 & 0.245 & 0.024 & 0.070 & 0.268 & 0.035 \\
\hline Mg I & 0.026 & 0.126 & 0.026 & 0.048 & 0.172 & 0.013 & 0.047 & 0.166 & 0.019 \\
\hline Al I & 0.033 & 0.149 & 0.029 & 0.057 & 0.214 & 0.021 & 0.067 & 0.264 & 0.038 \\
\hline $\mathrm{Si}$ I & 0.026 & 0.141 & 0.028 & 0.031 & 0.144 & 0.044 & 0.042 & 0.159 & 0.039 \\
\hline $\mathrm{Ca} \mathrm{I}$ & 0.029 & 0.129 & 0.032 & 0.044 & 0.193 & 0.047 & 0.031 & 0.128 & 0.070 \\
\hline Sc II & 0.032 & 0.141 & 0.034 & 0.047 & 0.195 & 0.041 & 0.065 & 0.355 & 0.046 \\
\hline Ti I & 0.033 & 0.151 & 0.035 & 0.034 & 0.116 & 0.056 & 0.037 & 0.117 & 0.046 \\
\hline Ti II & 0.029 & 0.135 & 0.035 & 0.044 & 0.190 & 0.057 & 0.043 & 0.175 & 0.043 \\
\hline $\mathrm{V}_{\mathrm{I}}$ & 0.035 & 0.155 & 0.033 & 0.062 & 0.268 & 0.072 & 0.049 & 0.166 & 0.045 \\
\hline Cr I & 0.026 & 0.121 & 0.038 & 0.045 & 0.207 & 0.058 & 0.053 & 0.192 & 0.076 \\
\hline Mn I & 0.028 & 0.130 & 0.036 & 0.044 & 0.173 & 0.036 & 0.053 & 0.160 & 0.058 \\
\hline $\mathrm{Fe} I$ & 0.026 & 0.108 & 0.035 & 0.040 & 0.162 & 0.050 & 0.043 & 0.192 & 0.062 \\
\hline $\mathrm{Fe}$ II & 0.028 & 0.125 & 0.035 & 0.045 & 0.170 & 0.039 & 0.061 & 0.309 & 0.046 \\
\hline Co I & 0.029 & 0.140 & 0.027 & 0.059 & 0.213 & 0.043 & 0.055 & 0.246 & 0.051 \\
\hline $\mathrm{Ni} \mathrm{I}$ & 0.027 & 0.128 & 0.031 & 0.039 & 0.147 & 0.051 & 0.056 & 0.254 & 0.068 \\
\hline $\mathrm{Cu} \mathrm{I}$ & 0.026 & 0.134 & 0.045 & 0.050 & 0.201 & 0.099 & 0.055 & 0.237 & 0.114 \\
\hline Y II & 0.040 & 0.180 & 0.042 & 0.065 & 0.245 & 0.069 & 0.115 & 0.557 & 0.056 \\
\hline Ba II & 0.038 & 0.179 & 0.044 & 0.079 & 0.305 & 0.033 & 0.089 & 0.417 & 0.056 \\
\hline La II & 0.042 & 0.154 & 0.098 & 0.031 & 0.074 & 0.049 & 0.037 & 0.092 & 0.044 \\
\hline Ce II & 0.044 & 0.129 & 0.145 & 0.021 & 0.051 & 0.087 & 0.017 & 0.042 & 0.162 \\
\hline $\mathrm{Nd}$ II & 0.050 & 0.229 & 0.061 & 0.097 & 0.394 & 0.086 & 0.083 & 0.287 & 0.075 \\
\hline Eu II & 0.020 & 0.049 & 0.110 & 0.022 & 0.049 & 0.095 & 0.025 & 0.063 & 0.179 \\
\hline
\end{tabular}

\begin{tabular}{lcc}
\hline \hline Element & $\sigma X_{\mathrm{L} 16}$ & $\sigma X_{\text {here }}$ \\
\hline $\mathrm{Na}$ I & 0.021 & 0.017 \\
$\mathrm{Mg}$ I & 0.035 & 0.024 \\
$\mathrm{Al} \mathrm{I}$ & 0.046 & 0.029 \\
$\mathrm{Si} \mathrm{I}$ & 0.023 & 0.025 \\
$\mathrm{Ca}$ I & 0.023 & 0.023 \\
$\mathrm{Ti}$ I & 0.029 & 0.029 \\
$\mathrm{Ti}$ II & 0.032 & 0.028 \\
$\mathrm{~V} \mathrm{I}$ & 0.026 & 0.037 \\
$\mathrm{Cr}$ I & 0.026 & 0.021 \\
$\mathrm{Mn}$ I & 0.026 & 0.031 \\
Fe I & 0.023 & 0.024 \\
$\mathrm{Co}$ I & 0.030 & 0.024 \\
$\mathrm{Ni}$ I & 0.028 & 0.023 \\
$\mathrm{Cu}$ I & 0.036 & 0.025 \\
$\mathrm{Ba}$ II & 0.031 & 0.037 \\
\hline
\end{tabular}

Notes. The dispersions were obtained using the subsample of stars of $\mathrm{L} 16\left(\sigma X_{\text {here }}\right)$ and their values $\left(\sigma X_{\mathrm{L} 16}\right)$.

than 0.02 dex, in general). We consider that this fact shows that our uncertainties are slightly overestimated. For comparison purposes, we also include the same computation for the subsample of 16 stars analyzed by L16 for the elements that are common to both studies. In this smaller range of atmospheric parameters corresponding to solar analogs, we obtain slightly lower abundance dispersions, which are similar to those of L16, ranging from 0.017 to 0.035 dex. For NGC 2632 and Ruprecht 147, both the quoted uncertainties and the found dispersions are larger than for the Hyades in most of the chemical species. We cannot clearly conclude that the clusters are inhomogeneous only based on the comparison of the uncertainties with the abundance dispersions.

We obtain strong correlations among different abundance pairs in the three clusters. We performed linear fits to each pair of element abundances by using a Bayesian outlier detector with a Markov chain Monte Carlo (MCMC), as explained in detail in Hogg et al. (2010). In brief, the methodology computes a linear regression with an objective datapoint rejection, which models the outlier distribution. The method infers the parameters of the linear fit together with the mean and variance of the distribution of outliers as well as the number of outliers at the same time. The model is run through $50000 \mathrm{MCMC}$ realizations, taking the maximum of the posterior distribution and the standard deviation as the best values of the slope, intercept, and their uncertainties. We did not attempt to perform the fits when the number of stars with abundances is smaller than 15 or when the uncertainties in the abundances are greater than 0.05 dex.

In Fig. 10 we plotted some examples of the differential element abundance pairs $\delta X_{1}$ vs. $\delta X_{2}$. The full matrix of differential abundances for the three clusters are found in Figs. B.3-B.5. We also overplotted the obtained fits. For the Hyades, we obtain statistically significant correlations (larger than $3 \sigma$ ) of all pairs of abundances that we analyze. Some elements with larger intrinsic uncertainties (e.g., $\mathrm{Ba}, \mathrm{Nd}, \mathrm{Cu}$ ) tend to present less significant correlations. For the other two clusters, we have a smaller sample of stars selected as most probable members and larger uncertainties in the abundance measures. The less significant fit is the one of Na I vs. MnI in NGC 2632, which has a posterior with two peaks. For Ruprecht 147, two fits involving Si I have lower significances than $3 \sigma$. In Fig. 10 we see no dependence of the differential abundance pairs with respect to $T_{\text {eff }}$, showing that all types of stars correlate in the same way.

For the Hyades, a large number of slopes were retrieved, so we computed the compatibility among all of them within the quoted uncertainties: (i) The fits involving $\mathrm{Cu}$ I and $\mathrm{Ba}$ II that have non-Gaussian posterior distributions tend to not be incompatible with the others. However, in these cases, uncertainties are not well represented by the standard deviation. (ii) In the case of Nd II, all the slopes are larger than $3 \sigma$ when compared to most of the other cases. We attribute this to the larger uncertainties of this element in comparison with the others, which makes the dispersion in its dimension increase, and thus increase the value of the slope. (iii) We find compatible slopes for all other pairs of elements. This indicates that the differences in star-by-star abundance are due to a zero-point difference in the overall metallicity among the stars in the cluster, with no variation depending on the chemical element.

\subsubsection{Comparison with synthetic data}

To better interpret our results, we generated a set of simulated spectra of stars in two cases: a chemically homogeneous cluster and an inhomogeneous one. In this way, we can see what we should expect in the case of chemical inhomogeneity and compare it with the results obtained for the observed spectra. 


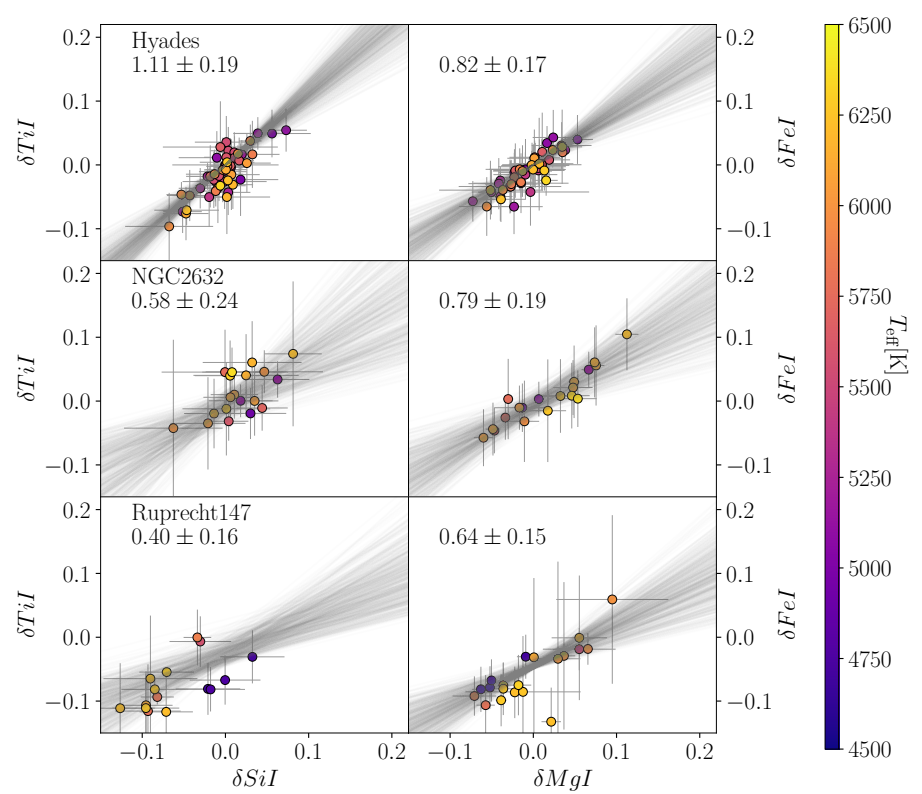

Fig. 10. Left: $\delta \mathrm{Ti}$ vs. $\delta \mathrm{Si}$. Right: $\delta \mathrm{Mg}$ vs. $\delta \mathrm{Fe}$, rows correspond to the three clusters. Color represents effective temperature. Translucid lines are the resulting linear fit according to the obtained posterior distribution in the intercept and the slope. The slope and its uncertainty is indicated in each panel.

For both the homogeneous and the inhomogeneous cases, we generated spectra of a set of stars with the same atmospheric parameters. We used parameters corresponding to the ones of two of the groups observed in the Hyades. That is, nine stars with $T_{\text {eff }}=6000-5850 \mathrm{~K}$ and $\log g=4.4-4.6 \mathrm{dex}$ as well as six stars with $T_{\text {eff }}=5850-5750 \mathrm{~K}$ and $\log g=4.4-4.6 \mathrm{dex}$. For the homogeneous case, we used a model metallicity of $[\mathrm{M} / \mathrm{H}]=0$ dex for all stars, whereas for the inhomogeneous case, we sampled a normal distribution with a mean metallicity of 0 dex and a standard deviation of 0.03 dex. We added Poissonian noise to the spectra to degrade the $\mathrm{S} / \mathrm{N}$ to between 100 and 200 . We analyzed both sets of spectra using the same procedure as for the real data.

Even if we introduce noise to the synthetic data, the experiment is not comparable to the analysis of real data. For example, the effects of the continuum normalization as well as bad or missing atomic data are not taken into account and can contribute to the dispersion of a synthetic homogeneous cluster. To be more realistic, we added some possible sources of systematic uncertainties. To generate the spectra, we used the radiative transfer code SYNTHE (Kurucz 1993) and the ATLAS9 (Castelli \& Kurucz 2004) atmospheric models, instead of SPECTRUM (Gray \& Corbally 1994) and MARCS (Gustafsson et al. 2008), which are used in the analysis pipeline. We slightly altered the line shapes by varying the macroturbulence parameter. In the analysis, $v_{\text {mac }}$ is determined by using the empirical relation given in Blanco-Cuaresma et al. (2014a), which depends on $T_{\text {eff }}, \log g$, and $[\mathrm{Fe} / \mathrm{H}]$. To generate the spectra, we added a random term $\left( \pm 0.15 \mathrm{~km} \mathrm{~s}^{-1}\right)$ to the estimated value.

We compared the recovered effective temperature and surface gravity with the input ones in the two cases, and we obtain almost the same small offsets on average (recovered - input): $\Delta T_{\text {eff }} \sim-16 \mathrm{~K}$ and $\Delta \log g \sim-0.03$. For $[\mathrm{M} / \mathrm{H}]$, the inhomogeneous case gives a larger offset and dispersion $(\Delta[\mathrm{M} / \mathrm{H}]=$ $-0.023 \pm 0.018$; standard deviation) than the homogeneous one $(-0.009 \pm 0.005)$.

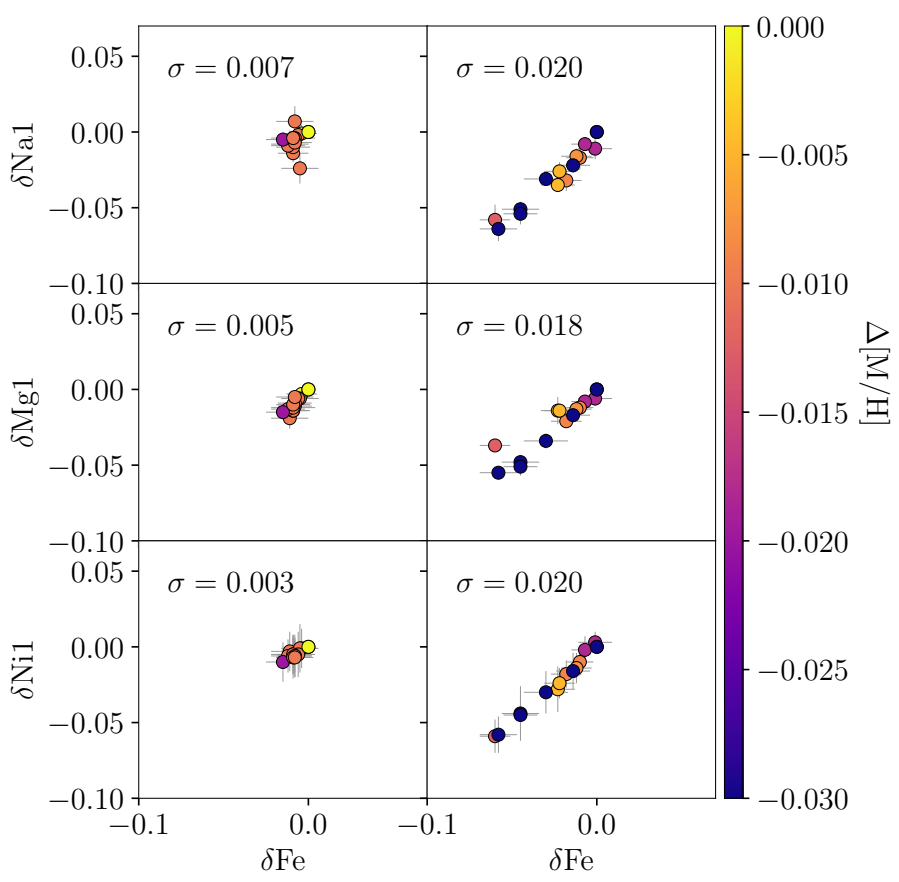

Fig. 11. Differential abundances of $\mathrm{Na}, \mathrm{Mg}$, and $\mathrm{Ni}$ as a function of $\mathrm{Fe}$ abundance for the simulated homogeneous (left) and inhomogeneous (right) OCs. The dispersions in the differential abundances are indicated in each panel. The points are colored according to the difference between the recovered and input metallicity.

We plotted three examples of the resulting differential abundances of the two cases as a function of the Fe differential abundance in Fig. 11. The overall abundance dispersions are one order of magnitude larger in the inhomogeneous case compared to the homogeneous one. Moreover, the dispersions in the homogeneous case are usually of the same order as the abundance uncertainties. Tight abundance correlations appear in the inhomogeneous cluster, and they appear for any given pair of chemical species, although we only show a few cases in the figure. For the homogeneous case, a hint of correlation is seen for some elements (e.g., $\mathrm{Mg}$ and $\mathrm{Ni}$ ), but it can be explained by the wrong choice of the metallicity of the model, indicated by the color of the points. This means that a slightly smaller abundance in all elements is expected if the chosen atmosphere model has a lower (and wrong) overall metallicity. On the contrary, in the inhomogeneous case, there is no indication that the retrieved smaller differential abundances are due to a wrong metallicity in the model.

Compared with the results of Fig. 10, the analyzed stars in the three clusters resemble the inhomogeneous case in all chemical species in which we have been able to perform a fit. We conclude that the analyzed OCs present signs of chemical inhomogeneity at a level of $\sim 0.02-0.03$ dex with no clear dependence on the chemical species.

\subsection{Chemical tagging}

Our sample of stars is well suited to investigate the chemical tagging using differential abundance computation. In Fig. 12 we plotted the weighted average abundances and dispersions for the following three groups of stars: $\mathrm{K}$ giants, $\mathrm{G} \operatorname{dwarfs}^{13}$, and $\mathrm{F}$ dwarfs. We only included elements that we were able to measure in at least one star in the three groups and the three clusters.

${ }^{13}$ We restrict this group to $5900>T_{\text {eff }}>5500 \mathrm{~K}$ since only the Hyades have a representative number of stars cooler than that. 


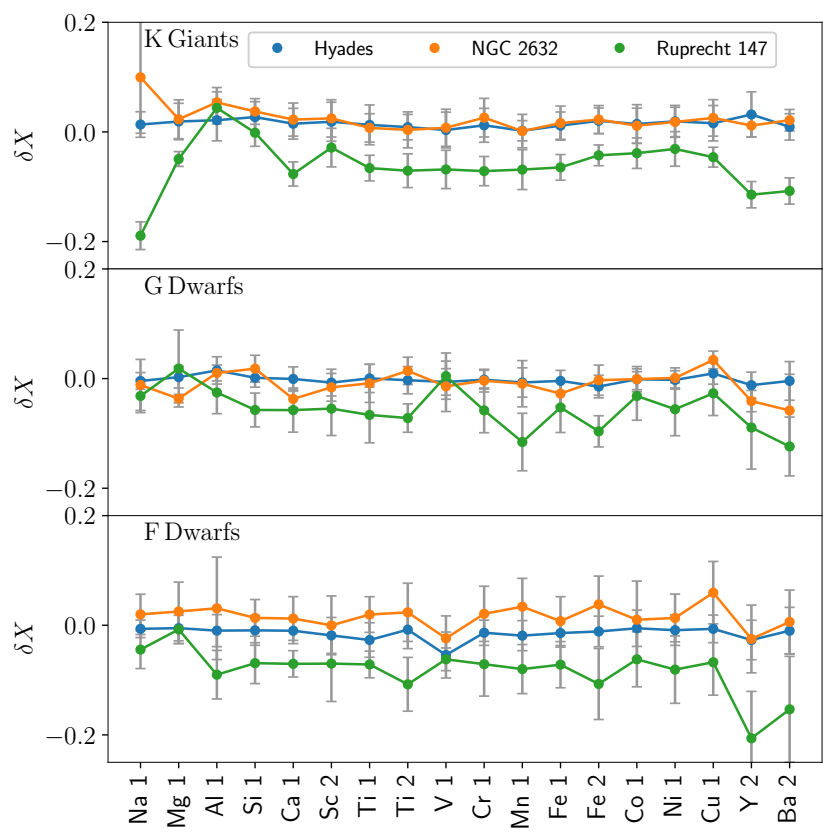

Fig. 12. Differential chemical abundances (weighted average) of all analyzed elements of: $\mathrm{K}$ giants (top), $\mathrm{G}$ dwarfs (middle), and $\mathrm{F}$ dwarfs (bottom).

G dwarfs and K giants in NGC 2632 and the Hyades have an equivalent chemical signature for almost all elements. In terms of the chemical evolution of the Galaxy, this is consistent with the two OCs having similar ages, as computed in the literature on several occasions using isochrones. However, some discrepancies are still found in the age value; they are usually quoted between 600 and 800 Myr depending on methodology and models used (e.g., Brandt \& Huang 2015; Gossage et al. 2018).

Instead, the F dwarfs group for NGC 2632 appears enhanced in all elements compared to the Hyades stars, but with large dispersions. This is mainly produced by few stars in this cluster in the range of $6000<T_{\text {eff }}<6200 \mathrm{~K}$. They give very consistent abundances among them, but they are $\sim 0.05$ dex larger than the other stars in the F dwarfs group, the latter matching the abundances of the Hyades. We have checked that this is not due to a low $\mathrm{S} / \mathrm{N}$ of the spectra, or to a particular instrument configuration, and also does not depend on the choice of the reference star. This effect can be related to enhancement by the convection of stars near the turnoff since the mass of the convective zone increases at this temperature range (Pinsonneault et al. 2001). Another possibility is that this is caused by atomic diffusion for which some models predict a dip in the abundances in this region of the HR diagram (e.g., Souto et al. 2019). Further investigation is needed to reach a conclusion.

In the three panels, we can differentiate the chemical signature of Ruprecht 147 in comparison with the other two clusters, where the abundances for Ruprecht 147 are systematically lower by typically $0.05-0.07 \mathrm{dex}$. In the case of giants, uncertainties are lower so this allows for one to better differentiate between the clusters. This chemical separation is explained with the different age of this cluster, around 2.5 Gyr (see Table 1). We note the particular case of lower Na I for the giants in Ruprecht 147 (compared to the Hyades). We attribute this as an effect of internal mixing in the surface of massive giants described by the stellar evolutionary models of Lagarde et al. (2012), for instance. Similar Na differences were identified in Smiljanic et al. (2016) for red clump stars in clusters depending on stellar mass, where stars more massive than $\sim 2 M_{\odot}$ can present overabundances up

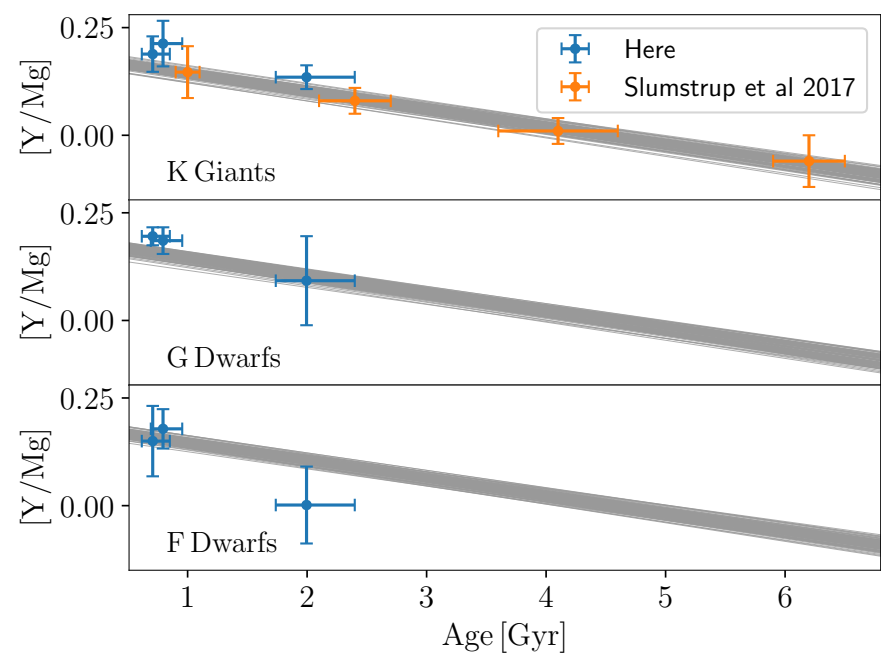

Fig. 13. $[\mathrm{Y} / \mathrm{Mg}]$ abundances with respect to the age of the three clusters studied in this work (blue) and the clusters studied in Slumstrup et al. (2017) (orange). We plotted the results of the different spectral types in the three planets. We overplotted the relation found by Tucci Maia et al. (2016) in gray.

to 0.2 dex. Ruprecht 147 is much older than the Hyades, therefore, its giants are less massive; when checking with isochrones, they are about $1.6 M_{\odot}$, compared to $2.5 M_{\odot}$ in the Hyades. So in our case, we see $\mathrm{Na}$ underabundances since we differentially analyze with respect to the Hyades. Non-LTE effects could also play a role in our analysis since the giants in Ruprecht 147 have slightly different atmospheric parameters with respect to the Hyades.

We find peculiarities in several elements, for example in Y II and Ba II, which differ in Ruprecht 147 compared to the other two clusters. This is probably due to the different nucleosynthetic origin of these two elements, which are basically produced by neutron captures via the $s$-process. The impact of the age on the chemical abundances of these types of elements motivates their use to construct "chemical clocks" (e.g., Tucci Maia et al. 2016). In Fig. 13 we plotted the derived [Y/Mg] as a function of the age reported by Gaia Collaboration (2018a). We computed $[\mathrm{Y} / \mathrm{Mg}]$ from our differential abundances with respect to the Hyades, and we added the absolute $[\mathrm{Y} / \mathrm{H}]$ and $[\mathrm{Mg} / \mathrm{H}]$ abundance of the Hyades with respect to the Sun (Table 4). Our uncertainties for Ruprecht 147 dwarfs are quite large for these two elements, mainly because the $\mathrm{S} / \mathrm{N}$ are the lowest for these spectra. For comparison purposes, we overplotted the empirical relation derived by Tucci Maia et al. (2016) using solar twins by taking into account the uncertainties that they quote. We added the four clusters that were analyzed by Slumstrup et al. (2017) to the plot, where they computed the [Y/Mg] of one to three red giant stars in each cluster. The points of both studies are compatible with the empirical relation at $1 \sigma$. This result is interesting in the context of Galactic archaeology since it proves that helium-core burning giants follow this relation similarly to dwarfs.

\subsection{Tidal tails}

In our initial sample, we included ten and three stars from the tidal tails of the Hyades and NGC 2632, respectively. Several of them were removed from the sample in the membership refinement in Sect. 4. We have recovered the differential abundances 


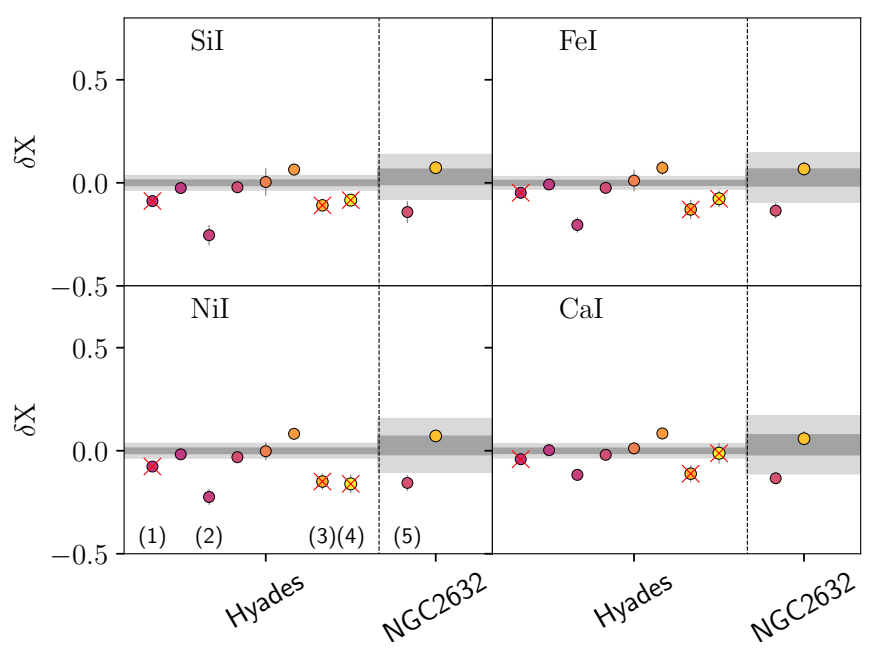

Fig. 14. Differential chemical abundances of $\mathrm{Si}, \mathrm{Fe}, \mathrm{Ni}$, and $\mathrm{Ca}$ of the analyzed stars in the tidal tails of the Hyades and NGC 2632. The color code is the same as in Fig. 7. Red crosses indicate stars identified as kinematic outliers. Shadowed regions represent the cluster signature: $1 \sigma$ (dark gray) and $3 \sigma$ (light gray) dispersion. Numbered stars are discussed in the text.

of those that could be analyzed differentially ${ }^{14}$, as indicated in Fig. 6, to compare their chemical signature with respect to the cluster.

We are able to compute differential abundances for eight stars in the Hyades and two in NGC 2632. As an example, we plotted in Fig. 14 abundances of Fe I, Si I, Ni I, and Mg I of the tidal tails stars, highlighting those rejected as outliers with crosses. The numbered stars in the plot are discussed below:

- The stars 2 and 5 , are out of the $3 \sigma$ signature of the respective clusters in almost all the chemical species. These were not identified as outliers in kinematics in Sect. 4, but they are probably not members.

- Star 3 is out of $3 \sigma$ in ten of the chemical species, and in the other cases is out of $1 \sigma$. It was identified as an outlier and it is probably a nonmember.

- Star 4 is out of $3 \sigma$ in seven elements and in most of the other elements, it is out of $1 \sigma$. It was identified as an outlier and can be a non-member.

- Star 1 is inside of $3 \sigma$ in most of the elements. It was identified as an outlier in Sect. 4 but the chemical signature is mainly compatible. It could be that this star is simply in the tail of the kinematic distribution of the cluster and that is why we rejected it as an outlier.

According to the results, merging kinematics, and chemistry, we have discarded as members, four (stars 2, 3, 4, and $5)$ out of ten analyzed stars. So we conclude that there exists significant contamination when selecting member stars in the outskirts of the clusters using only kinematics. This can be disentangled when looking at the detailed chemistry using differential analysis.s

\section{Conclusions}

In this work, we derived radial velocities, atmospheric parameters, and absolute and differential chemical abundances of 22 chemical species for stars in three nearby OCs: the Hyades, NGC 2632 (Praesepe), and Ruprecht 147. We investigated the

\footnotetext{
14 Three tidal tail stars rejected as outliers could not be analyzed differentially because they fall out of the group limits.
}

possibilities of differential analysis in order to analyze the homogeneity of nearby clusters and their tidal tails as well as the chemical tagging using stars in different evolutionary states. We used the most recent membership of the three clusters, which are very accurate thanks to Gaia DR2 exquisite astrometry at small distances $(d<300 \mathrm{pc})$. We queried the public archives to look for high resolution and high $\mathrm{S} / \mathrm{N}$ spectra for the targeted stars, and we also performed our own observational programs. We were able to analyze spectra of 62,22 , and 24 stars in the Hyades, NGC 2632, and Ruprecht 147, respectively.

To ensure the sample of stars did not have contaminants, we did a membership refinement computing total Galactic velocities from the Gaia DR2 proper motions and parallaxes, and the obtained radial velocities from our pipeline. We rejected several outliers previously classified as members, which can be contaminants, spectroscopic binaries, or stars in the tails of the Galactic velocity distribution. We obtained atmospheric parameters and chemical abundances with respect to the Sun $([\mathrm{X} / \mathrm{H}])$. We show that bracket abundances depend on the atmospheric parameters for certain elements (e.g., $\mathrm{Na}, \mathrm{Mg}, \mathrm{V}, \mathrm{Mg}$, and La). We attribute this to a mix of non-LTE effects, physical changes of the abundances in the stellar atmosphere depending on the evolutionary stage, and systematics in the analysis.

Strictly line-by-line differential abundances were computed for a sample of 92 stars in eight groups of twin stars, using a star from the Hyades as a reference. The precision of the derived differential abundances was between 0.01 and $0.02 \mathrm{dex}$, with the exception of the heavy elements. A great effort was made to quantify the uncertainties of the analysis, internally and externally, following the recommendations of Jofré et al. (2019). We compared the results of different spectra of the same star that was analyzed independently, we tested the effects of the uncertainties in the atmospheric parameters, and we performed an extensive comparison of the stellar parameters and abundances retrieved for the GBS.

Differential abundances did not present a dependence with spectral type. An exception to this is Ruprecht 147 giants, which showed underabundances of $\mathrm{Na}$ of almost 0.2 dex with respect to the Hyades. Several studies propose that red giants that are more massive than $2 M_{\odot}$ can present $\mathrm{Na}$ overabundances due to internal mixing (e.g., Lagarde et al. 2012). Because of the age difference of the Hyades and Ruprecht 147, their giants have $\sim 2.5 M_{\odot}$ and $\sim 1.6 M_{\odot}$, and so this is consistent with the found difference.

We investigated the level of chemical homogeneity of the three clusters using the set of differential abundances obtained after combining different kinds of stars and instruments. We obtain large amplitudes in all chemical species, compared with our uncertainties, and dispersions of the order 0.02-0.03 dex in the Hyades. Moreover, very significant correlations are found for almost all pairs of elements with low dispersion. We used our pipeline to analyze a homogeneous vs. inhomogeneous synthetic cluster, showing that correlations appear when stars have some level of chemical inhomogeneity. This confirms the analysis done previously by Liu et al. (2016a) for the Hyades, with three times more stars. Most of the slopes in the correlations between abundance pairs are found compatible and are around one, showing that this is due to an overall zero-point difference in the stars. For NGC 2632 and Ruprecht 147, we deal with larger uncertainties, especially for the dwarfs, which make the dispersions and amplitudes in abundances larger than those of the Hyades. We also obtain signs of abundance correlations in several abundance pairs, which suggests some chemical inhomogeneity also.

Chemical tagging was analyzed for three spectral types of stars: $\mathrm{F}$ dwarfs, $\mathrm{G}$ dwarfs, and $\mathrm{K}$ giants. A clear difference in 
the chemical signature is seen for Ruprecht 147 with respect to the other two clusters due to the different age ( $\sim 2.5 \mathrm{Gyr}$ vs. $\sim 700 \mathrm{Myr}$ of the Hyades). The largest difference is seen for the giants, where we benefit from lower uncertainties in the abundances. A particularly large difference in the signatures is found for the $s$-process elements Y and Ba. The Hyades and NGC 2632 are indistinguishable in almost all elements, which is consistent with the two clusters having the same age. We note the enhancement of about $\sim 0.05$ dex found for a few $\mathrm{F}$ dwarfs in the temperature range of $6000<T_{\text {eff }}<6200 \mathrm{~K}$. The effect needs further investigation, it can be related to the change of the convective zones and/or to atomic diffusion.

Finally, we analyzed, in detail, the differential chemical abundances of the stars identified in the tidal tails of the Hyades (eight stars) and NGC 2632 (two stars), with respect to the cluster chemical signature. Four out of the ten stars do not seem to be members, according to the chemistry. Two of them can be identified as outliers when analyzing total Galactic velocities using radial velocity coming from high-resolution spectroscopy. We conclude that a lot of contamination exists when selecting members in the outskirts of a cluster, based only on kinematics.

Acknowledgements. We gratefully thank the anonymous referee for providing comments that have improved the quality of the work. This work has made use of data from the European Space Agency (ESA) mission Gaia (http://www.cosmos.esa.int/gaia), processed by the Gaia Data Processing and Analysis Consortium (DPAC, http://www.cosmos.esa.int/web/ gaia/dpac/consortium). We acknowledge the Gaia Project Scientist Support Team and the Gaia DPAC. Funding for the DPAC has been provided by national institutions, in particular, the institutions participating in the Gaia Multilateral Agreement. This research made extensive use of the SIMBAD database, and the VizieR catalog access tool operated at the CDS, Strasbourg, France, and of NASA Astrophysics Data System Bibliographic Services. This research has made use of Astropy (Astropy Collaboration 2013), Topcat (Taylor 2005). L. C., C. S., and Y. T. acknowledge support from "programme national de physique stellaire" (PNPS) and from the "programme national cosmologie et galaxies" (PNCG) of CNRS/INSU. U. H. acknowledges support from the Swedish National Space Agency (SNSA/Rymdstyrelsen).

\section{References}

Astropy Collaboration (Robitaille, T. P., et al.) 2013, A\&A, 558, A33 Bergemann, M., Gallagher, A. J., Eitner, P., et al. 2019, A\&A, 631, A80 Blanco-Cuaresma, S. 2019, MNRAS, 486, 2075

Blanco-Cuaresma, S., \& Fraix-Burnet, D. 2018, A\&A, 618, A65

Blanco-Cuaresma, S., Soubiran, C., Heiter, U., \& Jofré, P. 2014a, A\&A, 569, A111

Blanco-Cuaresma, S., Soubiran, C., Jofré, P., \& Heiter, U. 2014b, A\&A, 566, A98

Boesgaard, A. M., Roper, B. W., \& Lum, M. G. 2013, ApJ, 775, 58

Bossini, D., Vallenari, A., Bragaglia, A., et al. 2019, A\&A, 623, A108

Bragaglia, A., Fu, X., Mucciarelli, A., Andreuzzi, G., \& Donati, P. 2018, A\&A, 619, A176

Brandt, T. D., \& Huang, C. X. 2015, ApJ, 807, 24

Bressan, A., Marigo, P., Girardi, L., et al. 2012, MNRAS, 427, 127

Cantat-Gaudin, T., Jordi, C., Vallenari, A., et al. 2018, A\&A, 618, A93

Carlberg, J. K. 2014, AJ, 147, 138

Casamiquela, L., Blanco-Cuaresma, S., Carrera, R., et al. 2019, MNRAS, 490, 1821
Castelli, F., \& Kurucz, R. L. 2004, ArXiv e-prints [arXiv:astro-ph/0405087] Curtis, J. L., Wolfgang, A., Wright, J. T., Brewer, J. M., \& Johnson, J. A. 2013, AJ, 145, 134

Dias, W. S., Alessi, B. S., Moitinho, A., \& Lépine, J. R. D. 2002, A\&A, 389, 871 Dotter, A., Conroy, C., Cargile, P., \& Asplund, M. 2017, ApJ, 840, 99

Friel, E. D., Janes, K. A., Tavarez, M., et al. 2002, AJ, 124, 2693

Gaia Collaboration (Babusiaux, C., et al.) 2018a, A\&A, 616, A10

Gaia Collaboration (Brown, A. G. A., et al.) 2018b, A\&A, 616, A1

Gebran, M., Vick, M., Monier, R., \& Fossati, L. 2010, A\&A, 523, A71

Gilmore, G., Randich, S., Asplund, M., et al. 2012, The Messenger, 147, 25

Gossage, S., Conroy, C., Dotter, A., et al. 2018, ApJ, 863, 67

Gray, R. O., \& Corbally, C. J. 1994, AJ, 107, 742

Grevesse, N., Asplund, M., \& Sauval, A. J. 2007, Space Sci. Rev., 130, 105

Gustafsson, B., Edvardsson, B., Eriksson, K., et al. 2008, A\&A, 486, 951

Hawkins, K., Masseron, T., Jofré, P., et al. 2016, A\&A, 594, A43

Heiter, U., \& Luck, R. E. 2003, AJ, 126, 2015

Heiter, U., Lind, K., Asplund, M., et al. 2015a, Phys. Scr., 90, 054010

Heiter, U., Jofré, P., Gustafsson, B., et al. 2015b, A\&A, 582, A49

Heiter, U., Lind, K., Bergemann, M., et al. 2019, A\&A, submitted

Hogg, D. W., Bovy, J., \& Lang, D. 2010, ArXiv e-prints [arXiv:1008.4686]

Jofré, P., Heiter, U., Soubiran, C., et al. 2014, A\&A, 564, A133

Jofré, P., Heiter, U., Soubiran, C., et al. 2015, A\&A, 582, A81

Jofré, P., Heiter, U., Tucci Maia, M., et al. 2018, Res. Notes Am. Astron. Soc., 2, 152

Jofré, P., Heiter, U., \& Soubiran, C. 2019, ARA\&A, 57, 571

Kharchenko, N. V., Piskunov, A. E., Röser, S., Schilbach, E., \& Scholz, R.-D. 2005, A\&A, 438, 1163

Kurucz, R. 1993, SYNTHE Spectrum Synthesis Programs and Line Data. Kurucz CD-ROM No. 18 (Cambridge, MA: Smithsonian Astrophysical Observatory)

Lagarde, N., Decressin, T., Charbonnel, C., et al. 2012, A\&A, 543, A108

Lind, K., Asplund, M., Barklem, P. S., \& Belyaev, A. K. 2011, A\&A, 528, A103

Liu, F., Yong, D., Asplund, M., Ramírez, I., \& Meléndez, J. 2016a, MNRAS, 457, 3934

Liu, F., Asplund, M., Yong, D., et al. 2016b, MNRAS, 463, 696

Liu, F., Asplund, M., Yong, D., et al. 2019, A\&A, 627, A117

Mahdi, D., Soubiran, C., Blanco-Cuaresma, S., \& Chemin, L. 2016, A\&A, 587, A131

Majewski, S. R., Schiavon, R. P., Frinchaboy, P. M., et al. 2017, AJ, 154, 94

Marigo, P., Girardi, L., Bressan, A., et al. 2017, ApJ, 835, 77

Meingast, S., \& Alves, J. 2019, A\&A, 621, L3

Meléndez, J., Asplund, M., Gustafsson, B., \& Yong, D. 2009, ApJ, 704, L66

Nielsen, M. B., Gizon, L., Schunker, H., \& Karoff, C. 2013, A\&A, 557, L10

Nissen, P. E. 2015, A\&A, 579, A52

Önehag, A., Gustafsson, B., \& Korn, A. 2014, A\&A, 562, A102

Pinsonneault, M. H., DePoy, D. L., \& Coffee, M. 2001, ApJ, 556, L59

Randich, S., Gilmore, G., \& Gaia-ESO Consortium 2013, The Messenger, 154, 47

Reggiani, H., Meléndez, J., Kobayashi, C., Karakas, A., \& Placco, V. 2017, A\&A, 608, A46

Riedel, A. R., Blunt, S. C., Lambrides, E. L., et al. 2017, AJ, 153, 95

Röser, S., \& Schilbach, E. 2019, A\&A, 627, A4

Röser, S., Schilbach, E., \& Goldman, B. 2019, A\&A, 621, L2

Slumstrup, D., Grundahl, F., Brogaard, K., et al. 2017, A\&A, 604, L8

Smiljanic, R., Romano, D., Bragaglia, A., et al. 2016, A\&A, 589, A115

Soubiran, C., Cantat-Gaudin, T., Romero-Gómez, M., et al. 2018, A\&A, 619, A155

Souto, D., Cunha, K., Smith, V. V., et al. 2018, ApJ, 857, 14

Souto, D., Allende Prieto, C., Cunha, K., et al. 2019, ApJ, 874, 97

Spina, L., Meléndez, J., Casey, A. R., Karakas, A. I., \& Tucci-Maia, M. 2018, ApJ, 863, 179

Taylor, M. B. 2005, in Astronomical Data Analysis Software and Systems XIV, eds. P. Shopbell, M. Britton, \& R. Ebert, ASP Conf. Ser., 347, 29

Tucci Maia, M., Ramírez, I., Meléndez, J., et al. 2016, A\&A, 590, A32

White, R. J., Gabor, J. M., \& Hillenbrand, L. A. 2007, AJ, 133, 2524

Yong, D., Meléndez, J., Grundahl, F., et al. 2013, MNRAS, 434, 3542 


\section{Appendix A: Tables}

Tables A.1 and A.2 include the retrieved abundances and their uncertainties of the sample GBS spectra (see main text), compared with the references for the available elements. Table A.3 includes the following for all cluster stars: star name, cluster name, number of spectra analyzed, radial velocity, Galactocentric velocity, $T_{\text {eff }}, \log g, v_{\text {mic }},[\mathrm{M} / \mathrm{H}]$, bracket, and differential abundances with errors. We include several flags that note any peculiarity found: (1) outlier Galactocentric velocity, (2) large radial velocity uncertainties, (3) star in the tidal tails, or (4) star used as a reference for differential abundance.

Table A.1. Detailed abundances of the GBS with respect to the Sun analyzed in this work (rows marked as "here") with the dispersion among the spectra quoted as uncertainty.

\begin{tabular}{|c|c|c|c|c|c|c|c|c|c|c|c|c|c|}
\hline Star & & Nspec & {$[\mathrm{Ca} \mathrm{I} / \mathrm{H}]$} & {$[\mathrm{Co} \mathrm{I} / \mathrm{H}]$} & {$[\mathrm{Cr} \mathrm{I} / \mathrm{H}]$} & {$[\mathrm{Fe} \mathrm{I} / \mathrm{H}]$} & {$[\mathrm{Mg} \mathrm{I} / \mathrm{H}]$} & {$[\mathrm{Mn} \mathrm{I} / \mathrm{H}]$} & {$[\mathrm{Ni} / \mathrm{H}]$} & {$[\mathrm{Sc} \mathrm{II} / \mathrm{H}]$} & {$[\mathrm{Si} \mathrm{I} / \mathrm{H}]$} & {$[\mathrm{Ti} \mathrm{I} / \mathrm{H}]$} & {$\left[\mathrm{V}_{\mathrm{I} / \mathrm{H}]}\right.$} \\
\hline $18 \mathrm{Sco}$ & here & 24 & $\begin{array}{r}0.04 \pm 0.01 \\
(0.03)\end{array}$ & $\begin{array}{r}0.02 \pm 0.01 \\
(0.03)\end{array}$ & $\begin{array}{r}0.04 \pm 0.01 \\
(0.03)\end{array}$ & $\begin{array}{r}0.03 \pm 0.01 \\
(0.03)\end{array}$ & $\begin{array}{r}0.02 \pm 0.01 \\
(0.01)\end{array}$ & $\begin{array}{r}0.02 \pm 0.01 \\
(0.05)\end{array}$ & $\begin{array}{r}0.02 \pm 0.01 \\
(0.04)\end{array}$ & $\begin{array}{r}0.03 \pm 0.01 \\
(0.02)\end{array}$ & $\begin{array}{r}0.03 \pm 0.01 \\
(0.04)\end{array}$ & $\begin{array}{r}0.02 \pm 0.01 \\
(0.04)\end{array}$ & $\begin{array}{r}0.01 \pm 0.01 \\
(0.04)\end{array}$ \\
\hline & Ref & & $0.06 \pm 0.06$ & $0.02 \pm 0.07$ & $0.05 \pm 0.06$ & $0.03 \pm 0.03$ & $0.04 \pm 0.05$ & $0.04 \pm 0.09$ & $0.04 \pm 0.07$ & $0.04 \pm 0.05$ & $0.05 \pm 0.03$ & $0.05 \pm 0.08$ & $0.04 \pm 0.07$ \\
\hline Arcturus & here & 5 & $\begin{array}{r}-0.55 \pm 0.02 \\
(0.07)\end{array}$ & $\begin{array}{r}-0.41 \pm 0.02 \\
(0.07)\end{array}$ & $\begin{array}{r}-0.58 \pm 0.01 \\
(0.07)\end{array}$ & $\begin{array}{r}-0.66 \pm 0.01 \\
(0.10)\end{array}$ & $\begin{array}{r}-0.26 \pm 0.01 \\
(0.04)\end{array}$ & $\begin{array}{r}-0.91 \pm 0.01 \\
(0.09)\end{array}$ & $\begin{array}{r}-0.59 \pm 0.01 \\
(0.08)\end{array}$ & $\begin{array}{r}-0.59 \pm 0.01 \\
(0.08)\end{array}$ & $\begin{array}{r}-0.37 \pm 0.02 \\
(0.06)\end{array}$ & $\begin{array}{r}-0.34 \pm 0.01 \\
(0.10)\end{array}$ & $\begin{array}{r}-0.34 \pm 0.02 \\
(0.07)\end{array}$ \\
\hline & Ref & & $-0.41 \pm 0.13$ & $-0.41 \pm 0.07$ & $-0.58 \pm 0.08$ & $-0.52 \pm 0.08$ & $-0.16 \pm 0.11$ & $-0.89 \pm 0.16$ & $-0.49 \pm 0.10$ & $-0.43 \pm 0.15$ & $-0.25 \pm 0.07$ & $-0.31 \pm 0.11$ & $-0.44 \pm 0.14$ \\
\hline
\end{tabular}

Notes. The number of used spectra fo each star is listed in the column Nspec. The median of the individual quoted uncertainties per spectra is indicated in parenthesis. In the rows marked as "Ref", we list the reference values of the GBS abundances. The full version of the table is available at the CDS.

Table A.2. Same as for Table A.1 for the elements for which we do not have a reference value.

\begin{tabular}{|c|c|c|c|c|c|c|c|c|c|c|c|c|}
\hline Star & Sspec & {$[\mathrm{Al} \mathrm{I} / \mathrm{H}]$} & {$[\mathrm{Ba} \mathrm{II} / \mathrm{H}]$} & {$[\mathrm{Ce} \mathrm{II} / \mathrm{H}]$} & {$[\mathrm{Cu} \mathrm{I} / \mathrm{H}]$} & {$[\mathrm{Eu} \mathrm{II} / \mathrm{H}]$} & {$[\mathrm{Fe} \mathrm{II} / \mathrm{H}]$} & {$[\mathrm{La} \mathrm{II} / \mathrm{H}]$} & {$[\mathrm{Na} / / \mathrm{H}]$} & {$[\mathrm{Nd} \mathrm{II} / \mathrm{H}]$} & {$[\mathrm{Ti} \mathrm{II} / \mathrm{H}]$} & {$[\mathrm{Y}$ II $/ \mathrm{H}]$} \\
\hline $18 \mathrm{Sco}$ & 24 & $\begin{array}{r}0.04 \pm 0.02 \\
(0.01)\end{array}$ & $\begin{array}{r}0.07 \pm 0.01 \\
(0.04)\end{array}$ & $\begin{array}{r}0.04 \pm 0.04 \\
(0.00)\end{array}$ & $\begin{array}{r}0.01 \pm 0.01 \\
(0.01)\end{array}$ & $\begin{array}{r}0.16 \pm 0.04 \\
(0.00)\end{array}$ & $\begin{array}{r}0.05 \pm 0.01 \\
(0.04)\end{array}$ & $\begin{array}{r}-0.01 \pm 0.03 \\
(0.00)\end{array}$ & $\begin{array}{r}0.02 \pm 0.01 \\
(0.05)\end{array}$ & $\begin{array}{r}0.06 \pm 0.02 \\
(0.12)\end{array}$ & $\begin{array}{r}0.04 \pm 0.01 \\
(0.04)\end{array}$ & $\begin{array}{r}0.09 \pm 0.01 \\
(0.03)\end{array}$ \\
\hline Arcturus & 5 & $\begin{array}{r}-0.23 \pm 0.03 \\
(0.01)\end{array}$ & $\begin{array}{r}-0.92 \pm 0.02 \\
(0.03)\end{array}$ & $\begin{array}{r}-0.77 \pm 0.04 \\
(0.00)\end{array}$ & $\begin{array}{r}-0.58 \pm 0.01 \\
(0.00)\end{array}$ & $\begin{array}{r}-0.39 \pm 0.03 \\
(0.00)\end{array}$ & $\begin{array}{r}-0.87 \pm 0.03 \\
(0.04)\end{array}$ & - & $\begin{array}{r}-0.44 \pm 0.02 \\
(0.02)\end{array}$ & $\begin{array}{r}-1.00 \pm 0.01 \\
(0.23)\end{array}$ & $\begin{array}{r}-0.47 \pm 0.03 \\
(0.08)\end{array}$ & $\begin{array}{r}-0.69 \pm 0.01 \\
(0.04)\end{array}$ \\
\hline
\end{tabular}

Notes. The full version of the table is available at the CDS.

Table A.3. Details of the cluster stars.

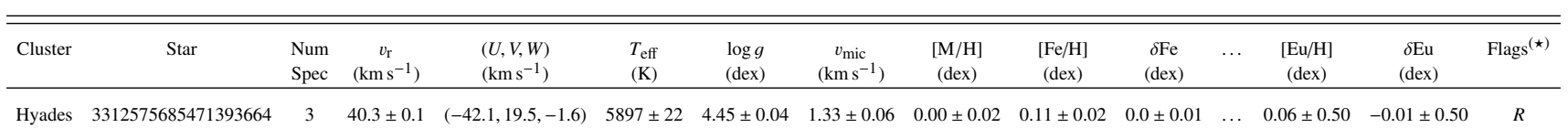

Notes. We list the cluster, Gaia DR2 source id, number of spectra analyzed, radial velocity, Galactic velocities, atmospheric parameters $\left(T_{\text {eff }}, \log g\right.$, $\left.v_{\text {mic }},[\mathrm{M} / \mathrm{H}]\right)$, and bracket and differential abundances with errors. We include several flags indicating any peculiarity found. ${ }^{(\star)}$ Flags: $\mathrm{O}=$ outlier in $(U, V, W) ; \mathrm{E}=$ large uncertainty in $v_{\mathrm{r}} ; \mathrm{T}=$ star in the tidal tails; $\mathrm{R}=$ star used as reference for the computation of differential abundances. The full version of the table is available at the CDS. 


\section{Appendix B: Additional figures}

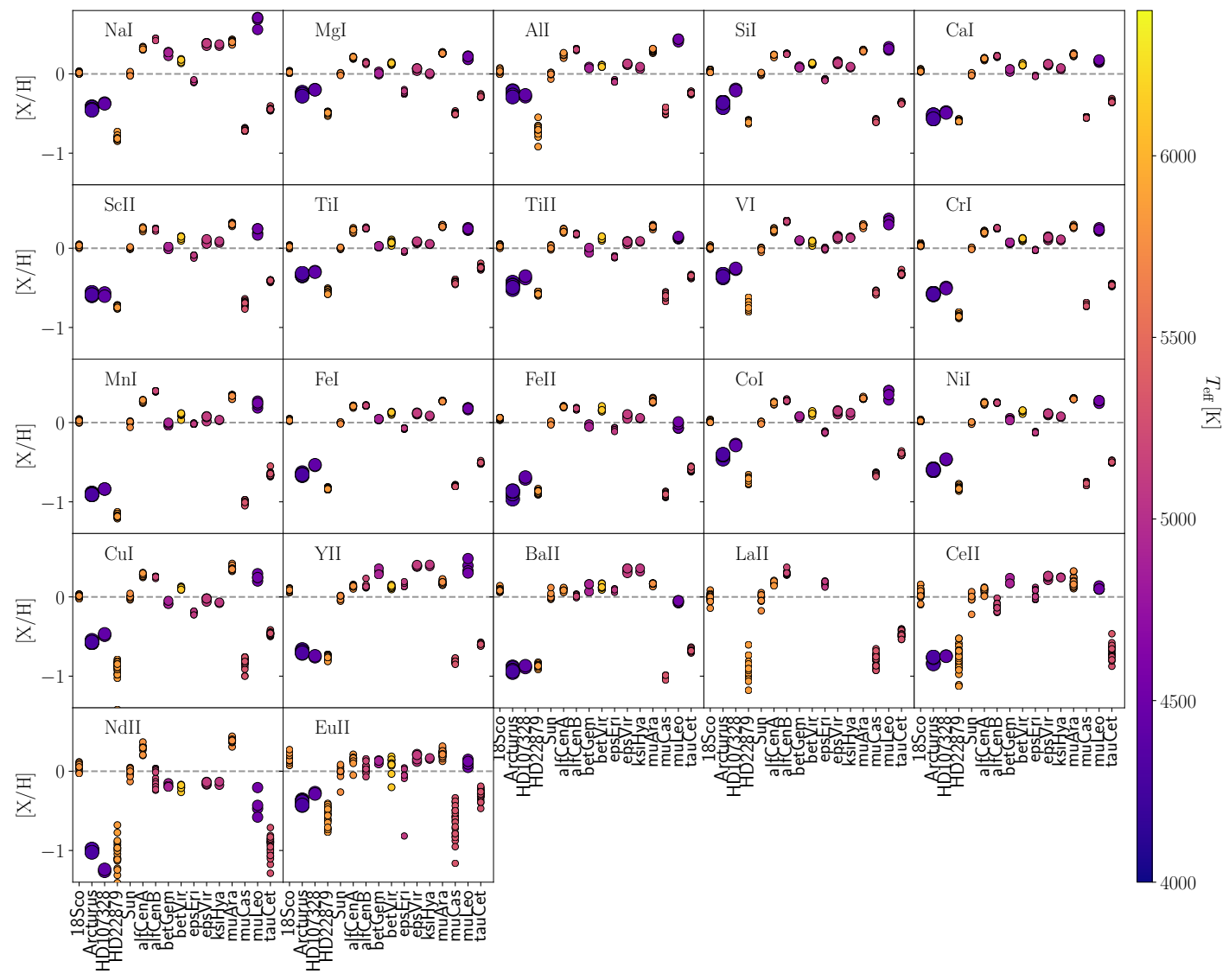

Fig. B.1. Abundances with respect to the mean solar abundance obtained in this work for the several spectra of the analyzed GBS (the stars are labeled by their names along the $x$-axis in alphabetical order). Color code corresponds to effective temperature, and symbol size is scaled by the surface gravity, where larger sizes correspond to giant stars (smaller gravity).

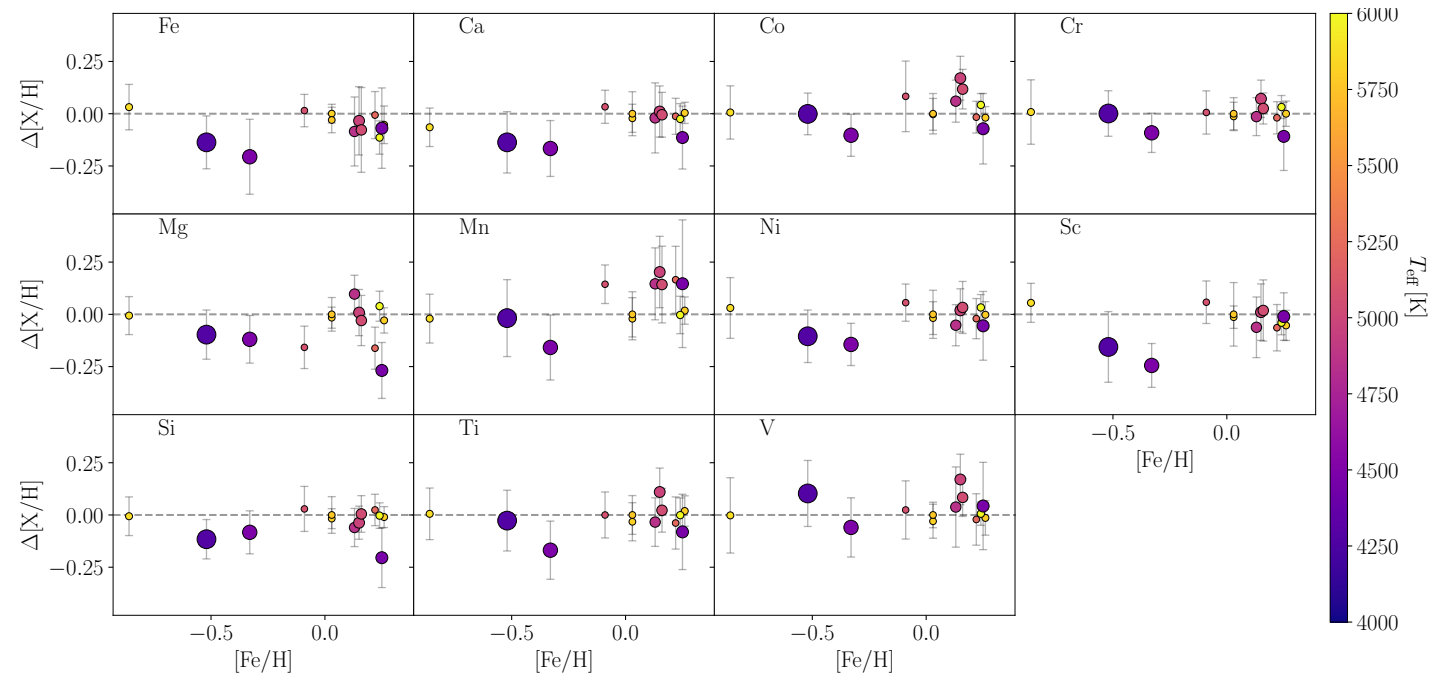

Fig. B.2. Differences (here - reference) in abundances $[\mathrm{X} / \mathrm{H}]$ for the selection of the GBS. We only plotted the elements for which a reference value exists in Jofré et al. (2015). Error bars correspond to the quadratic sum of the quoted errors in this work and in the reference. 


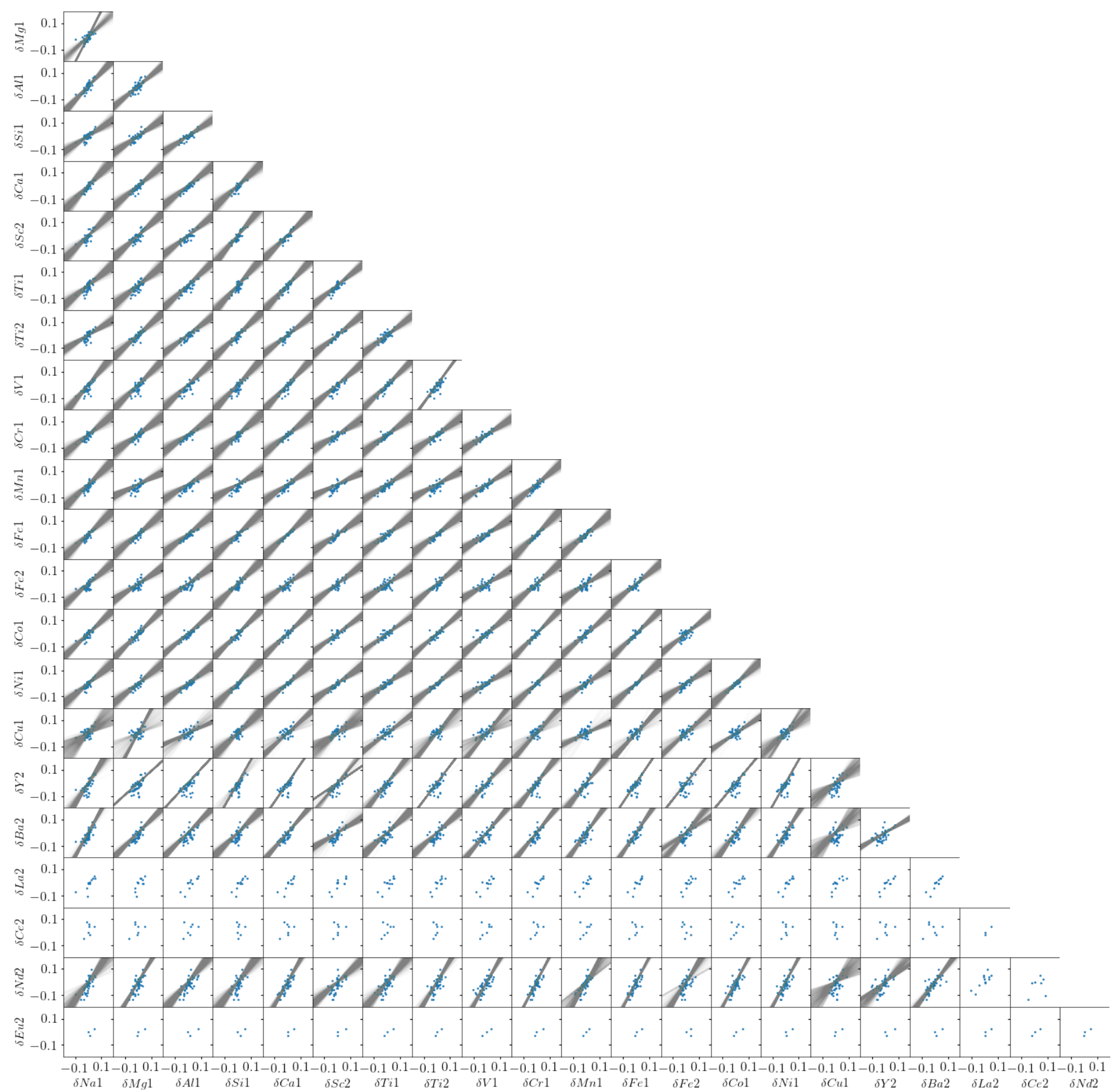

Fig. B.3. Differential abundances $\delta X$ and $\delta Y$ for all possible combination of elements in the Hyades stars. Gray lines represent the results and uncertainties of linear fits performed as described in the main text, sampling the posterior distribution. 
L. Casamiquela et al.: Differential abundances of nearby Open Clusters and their tidal tails

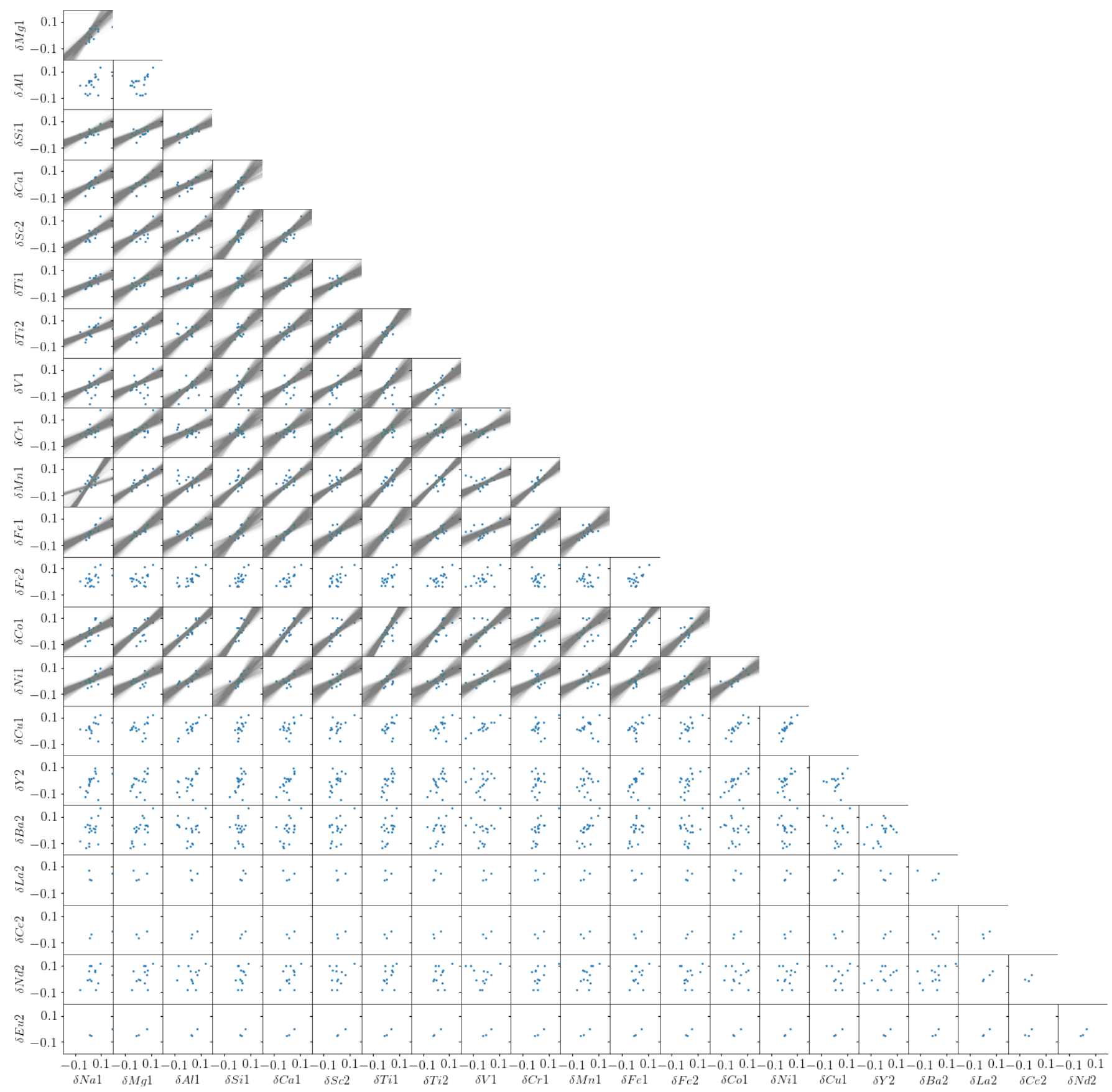

Fig. B.4. Same as Fig. B.3, but for NGC 2632. 


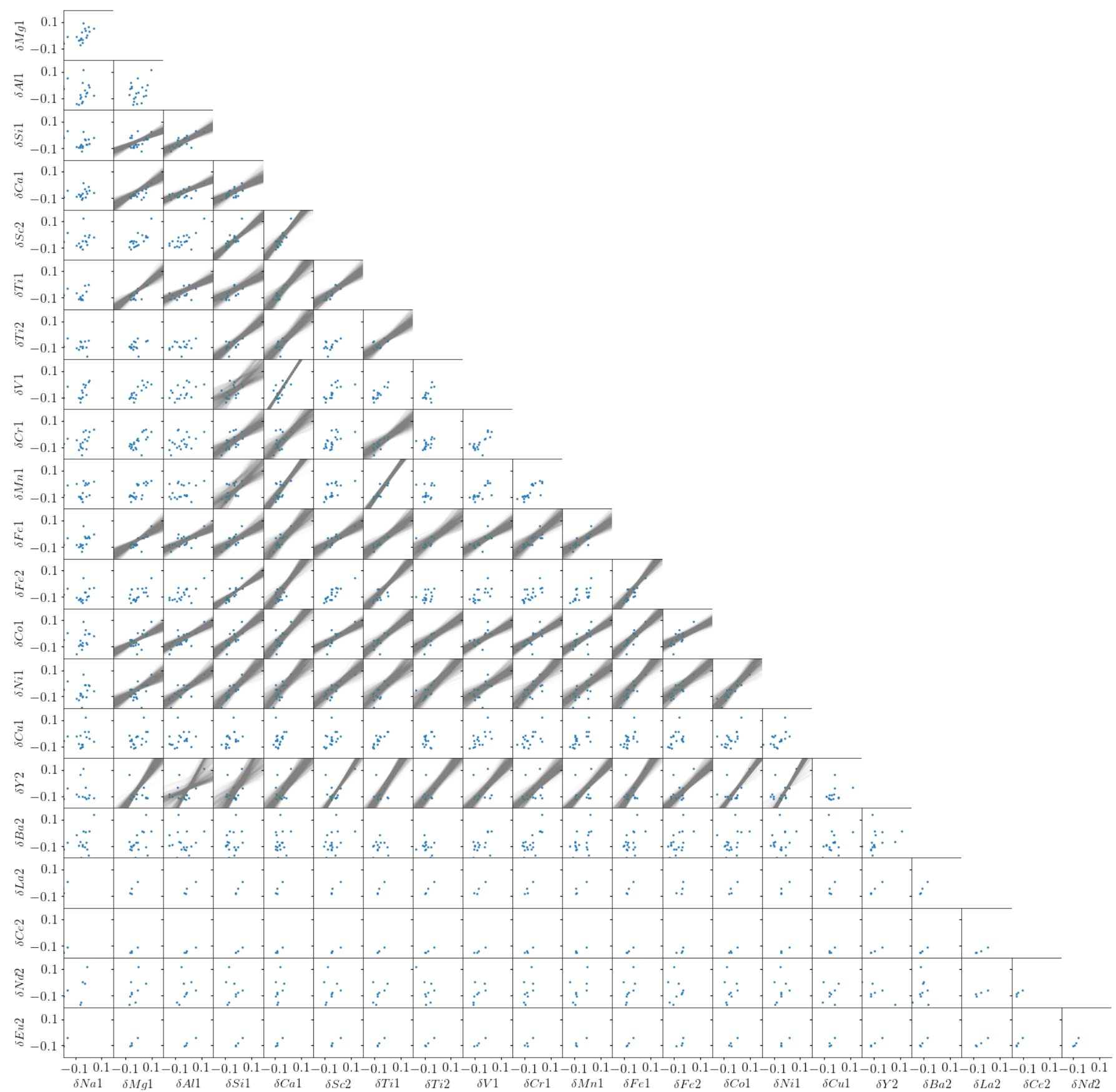

Fig. B.5. Same as Fig. B.3, but for Ruprecht 147. 